\title{
37. CARBONATE-FREE SEDIMENT COMPONENTS AND ASPECTS OF SILICA DIAGENESIS AT SITES 707, 709, AND 711 (LEG 115, WESTERN INDIAN OCEAN) ${ }^{1}$
}

\author{
Peter Hempel ${ }^{2}$ and Gerhard Bohrmann ${ }^{3}$
}

\begin{abstract}
From the equatorial Indian Ocean, carbonate-free portions of sediment samples of Paleocene to Miocene calcareous oozes and chalks from Sites 707, 709, and 711 were studied using X-ray diffraction measurements and the scanning electron microscope. Downhole variations in biogenic opal, quartz, barite, and clinoptilolite were investigated. The abundance patterns of these major mineral phases show several similarities and may be used for additional lithologic correlations.

Variations in biogenic opal contents reflect biogenic silica productivity. Beside the general pattern, a succession in biogenic silica decrease through time is generally recorded since the Oligocene. This succession started earliest at northernmost Site 711 and latest at southernmost Site 707, including Site 709 within these two. Opal-A variations as well as the barite distribution may be influenced by the paleoposition of the sites in relation to the high-productivity zone, which today lies south of the equator. Authigenic clinoptilolite apparently formed in two different modes. In deeper sediment intervals, clinoptilolite was the last mineral phase formed associated with enhanced silica diagenesis. In late Oligocene to middle Miocene sediments, clinoptilolite was the only authigenic silica phase encountered where otherwise strong opal dissolution was observed. The sponge spicules showed special dissolution features probably related to microbiological activity. Silica concretions mainly composed of opal-CT and authigenic quartz occur in carbonate-rich environments and are formed during later diagenesis when burial depth causes the sediments to reach higher temperatures. Opal-CT concretions in carbonate-free siliceous oozes were found at Site 711 and are probably formed during an early stage of silica diagenesis.
\end{abstract}

\section{INTRODUCTION}

Present pelagic sedimentation in the western equatorial Indian Ocean is dominated by calcareous skeletal particles, largely planktonic foraminifers, and calcareous nannoplankton. The major factors controlling deposition of these calcareous particles are the calcite compensation depth (CCD) and the productivity pattern. In this part of the ocean, the present CCD occurs at about $5200 \mathrm{~m}$ below sea level (mbsl), which causes good preservation of carbonate particles and the formation of calcareous oozes (Berger, 1976; Kolla et al., 1976; Van Andel, 1975).

For the first time during Ocean Drilling Program (ODP) Leg 115 , we recovered largely undisturbed sediment cores at almost $100 \%$ recovery rates from the Indian Ocean with the advanced piston corer (APC) system of the JOIDES Resolution. One of the major objectives of Leg 115 was to study changes in the CCD level through time. Such changes reflect important variabilities in the carbonate system and provide new insights into the paleoceanographic evolution of the Indian Ocean (Peterson and Backman, this volume). For reconstruction of the carbonate system during Cenozoic times, five sites (Sites 707 through 711) of a bathymetric south/north transect between $7^{\circ} 30^{\prime} \mathrm{S}$ to $2^{\circ} 45^{\prime} \mathrm{S}$ were drilled (Backman, Duncan, et al., 1988). The sites are situated in a narrow geographic area and cover a range of water depths between 1541 and $4428 \mathrm{~m}$, which represents the range of intermediate-, deep-, and bottom-water masses in this area (Warren, 1981). The majority of sediments drilled on the transect in the western Indian Ocean are calcareous oozes and

\footnotetext{
${ }^{1}$ Duncan, R. A., Backman, J., Peterson, L. C., et al., 1990. Proc. ODP, Sci. Results, 115: College Station, TX (Ocean Drilling Program).

2 GEOMAR, Forschungszentrum für marine Geowissenschaften and der Christian-Albrechts-Universität zu Kiel, Wischhofstrasse 1-3, Gebäude 4, D-2300 Kiel 14, Federal Republic of Germany.

3 Alfred-Wegener-Institut für Polar- und Meeresforschung (AWI), Columbusstrasse, D-2850 Bremerhaven, Federal Republic of Germany.
}

chalks with uniformly high carbonate contents $(80 \%-95 \%$; Backman, Duncan, et al., 1988); therefore, most sedimentologic and paleoceanographic studies are concentrated on the reconstruction of the carbonate system (e.g., Peterson and Backman, this volume).

To supplement these investigations, we focused our studies on the carbonate-free sediment fraction of three sites $(707,709$, and 711) of the transect. The carbonate-free portion in most of the drilled sediments represents only $5 \%-20 \%$ of the bulk material but nevertheless yields important information on sediment supply, depositional history, and diagenetic evolution of the noncarbonate facies. In particular, the aim of this study was to evaluate the occurrence and amount of detrital, biogenous pelagic, and authigenic constituents to recognize the evolution of this sedimentary facies. We focused our investigations on the non-clay minerals revealed by X-ray diffraction (XRD), especially biogenic silica and its diagenetic derivatives. The relationship between silica preservation and pore-water chemistry was investigated by Mikkelsen and Swart (this volume) and clay mineral studies were carried out by Failla et al. (this volume).

Biogenic opal (opal-A) dissolves to a large extent in the marine environment (Broecker and Peng, 1982; Calvert, 1983). In contrast to the carbonate system, an oceanwide saturation/dissolution boundary does not exist for biogenic silica as all ocean water is mostly undersaturated with respect to opal-A (Calvert, 1983). Special zones of enhanced opal dissolution develop in the uppermost part of the water column, where an intensive remineralization of opal skeletons occurs and at the sediment/ water interface, including the first centimeters of the sediment (Schrader and Schuette, 1981). In all zones, enhanced dissolution is a consequence of prolonged residence time and, hence, exposed to undersaturated seawater. The rate of dissolution mainly depends upon the sedimentation rate, that is, on how fast the biogenic silica is buried and therefore passes the corrosive zone at shallow sediment depths (Bohrmann, 1986). At greater burial depths and higher temperatures, biogenic silica dissolves and reprecipitates as opal-CT (Hein et al., 1978; Riech and von Rad, 
1979; Williams and Crerar, 1985, Williams et al., 1985). The generation of authigenic clinoptilolite is reported to be occasionally associated with silica diagenesis (Kastner, 1981; Riech and von Rad, 1979).

\section{MATERIAL AND METHODS}

Mineralogical data were obtained on a set of 165 selected samples by X-ray diffraction (XRD). Samples were treated with hydrogen peroxide and acetic acid to remove both organic matter and carbonate. Carbonate-free samples were mechanically ground and mixed with an internal standard of corundum $(\alpha-$ $\mathrm{Al}_{2} \mathrm{O}_{3}$ ) at a ratio of $2: 1$. Further grinding in an agate vial with acetone enhanced homogenization. Samples were then prepared as randomly oriented, pressed-powder slides. XRD-measurements were carried out using a Philips PW 1729 generator and $\mathrm{Co}_{\mathrm{K} \alpha}$ radiation. To quantify the opal bulge with high precision, the goniometer velocity was reduced to $0.005^{\circ} 2 \theta / \mathrm{s}$ and scans were run between $2^{\circ}-50^{\circ} 2 \theta$. Data processing was conducted using APD-1700 software (Philips) on a Micro PDP 11 computer system.

For the quantification of quartz, the ratio of $\mathrm{d}(101)$ of the quartz peak height (at $3.343 \AA$ ) to the $\mathrm{d}(012)$ corundum peak height (at $3.479 \AA$ ) was used. The absolute amount of quartz was then estimated from a standard curve of 11 mixtures of different amounts of quartz with a monomineralic matrix of smectite.

For measuring the content of biogenic opal, we primarily used the method of Eisma and Van der Gaast (1971). We graphically measured the height of the opal bulge between $17^{\circ}$ and $38^{\circ}$ $2 \theta \mathrm{Co}_{\mathrm{K} \alpha}$. The ratio of bulge height to the peak high of the corundum standard using (012) d-spacing (at $3.479 \AA$ ) was then calculated. The content of biogenic opal was finally estimated by comparing the ratio to a calibration curve based on several mixtures of recent opaline sponge spicules with a smectite matrix. A general calibration curve for clinoptilolite could be established, because of its wide range in chemical composition. This variation results in strong differences in d-spacings and relative intensities for selected X-ray reflections (Boles, 1972). Therefore, only the ratio of the intensity of the $(020)$ clinoptilolite dspacing to the intensity of the internal standard is used, keeping in mind, however, that d-spacing (9.054-8.970 $\AA$ ) intensity varies between $57 \%$ and $100 \%$ (Boles, 1972).

Barite was also quantified by the peak height ratio of d(121)spacing (at $3.103 \AA$ ) to that of the internal standard.

Untreated sediment samples, as well as carbonate-free samples, taken from selected intervals, were mounted for scanning electron microscopy (SEM). The SEM of the Geological Institute, Kiel (S 150 Cambridge), was used at $10 \mathrm{kV}$ connected to an energy-dispersive X-ray spectrographic analyzer (EDAX). In several intervals thin sections of cemented layers were studied by light microscopy.

\section{Regional Settings and Lithologic Sequences}

Site 707 (1541.4 mbsl) is the shallowest site of the transect and is located on the Mascarene Plateau between the Seychelles and Saya de Malha banks at $7^{\circ} 32.72^{\prime} \mathrm{S}, 59^{\circ} 01.01^{\prime} \mathrm{E}$. The northwestern part of the Mascarene Plateau separates the deep Arabian Sea Basin to the north from the Mascarene Basin to the south (Fig. 1). A sediment sequence of about $360 \mathrm{~m}$ overlies the igneous basement (Fig. 2).

Site 709 is located at $3^{\circ} 54.9^{\prime} \mathrm{S}, 60^{\circ} 33.1^{\prime} \mathrm{E}$ in a water depth of $3038.2 \mathrm{~m}$ (Fig. 1). The site lies in a small basin perched near the summit of the Madingley Rise, a regional topographic high between the Carlsberg Ridge and the Northern Mascarene Plateau. The irregular basement topography is draped with $50-$ $400 \mathrm{~m}$ of sediments. Site 709 was placed on top of the thicker se- quences to meet the primary objective of studying the continuous Neogene history of carbonate preservation (Fig. 2).

Site 711 lies on the northern edge of the Madingley Rise just a few hundred meters above the abyssal plain that separates the Madingley Rise from the Carlsberg Ridge, at $2^{\circ} 44.56^{\prime} \mathrm{S}$ and $61^{\circ} 09.78^{\prime} \mathrm{E}$, in a water depth of $4428.2 \mathrm{~m}$. It forms the deep end-member of the bathymetric transect (Fig. 2).

All three sites investigated are under the influence of the pelagic and hemipelagic depositional environment of the western tropical Indian Ocean. Biostratigraphic results indicate the recovery of more or less complete and continuous stratigraphic sections at all locations (Backman, Duncan, et al., 1988).

The sediments at Site 707 were divided into four units given their lithologic compositions (Fig. 3). Unit I (0-151 mbsf) consists of nannofossil-bearing foraminifer oozes and foraminifernannofossil oozes of Pleistocene to late Oligocene age. Condensed sequences characterize the latest Oligocene through middle Miocene interval, which probably implies that this interval contains several hiatuses of shorter duration. Unit II (151-212 mbsf) consists of late Oligocene to middle Eocene nannofossil oozes and chalks. The radiolarian-bearing nannofossil chalk and chert of Unit III (212-280 mbsf) are of middle Eocene age, and Unit IV (280-358 mbsf) comprises a sequence of middle Eocene to upper Paleocene interbedded carbonate lithotypes (Backman, Duncan, et al., 1988). These upper Paleocene and lower Eocene sediments are indicative of a shallow depositional environment. Microfossils of earliest upper Paleocene age show evidence for deposition in extremely shallow waters.

The first abundance of biosiliceous components occurs within Unit I, diminishing toward the base of Unit I and increasing again in Unit II. They remain as a minor sediment component throughout Units II and III. The transition from Unit III to IV is marked by the first onset of chert (Backman, Duncan, et al., 1988; Fig. 3) and partly silicified limestone.

The carbonate content of the sedimentary section of Site 709 , only occasionally falling below $80 \%$, indicates that the sediments at this site were always deposited above the CCD. Lower carbonate contents in the middle Eocene are probably caused by a larger input (dilution) of siliceous material rather than dissolution (Fig. 4). This is evident from the good preservation of the remaining calcareous nannofossils (Backman, Duncan, et al., 1988). Because of the homogeneous nature of the nannofossil ooze and chalk sequence, three subunits were defined on the basis of color changes and occurrence of abundant laminae (Backman, Duncan, et al., 1988). Biosiliceous components, mainly radiolarians, occur within Subunits IA and IB and in the lower part of Subunit IC.

Site 711 is characterized by a prominent shift from a mainly low carbonate content in the early Miocene to Pleistocene sediments of Units I and II to carbonate-rich sediments of the Paleogene (Units III and IV). This shift documents the passage of Site 711 from above the CCD in Paleogene times to below the $\mathrm{CCD}$ in the Neogene.

Intervals of high carbonate values within Units I and II are associated with calcareous turbidites that originated from the elevated parts of Madingley Rise. In Cores 115-711A-19X and $-20 \mathrm{X}$ (Fig. 5) of Unit IV, there are sections where the deposition of carbonate is replaced by clay-bearing radiolarian oozes and chalk (Backman, Duncan, et al., 1988). Only Unit II and the upper part of Unit III are barren of biosiliceous components.

\section{RESULTS}

Based on the XRD reflection records of the carbonate-free samples, we observed several minerals. Clay minerals and opal$\mathrm{A}$ are the most dominant noncarbonate mineral groups in the sediment sequences, with pyrite and feldspar only sporadically 


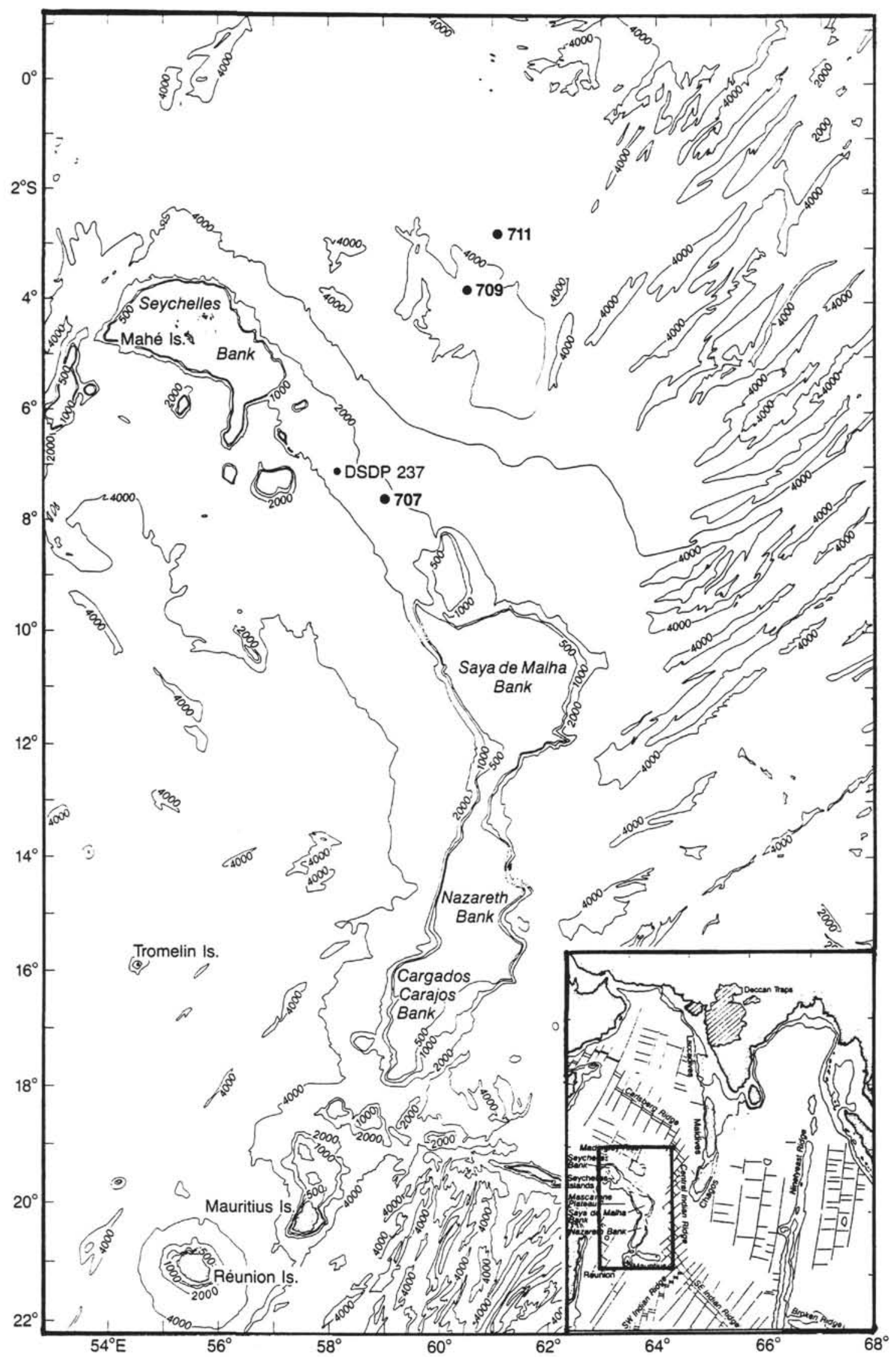

Figure 1. Location of Sites 707, 709, and 711 on Leg 115 in the western Indian Ocean. 

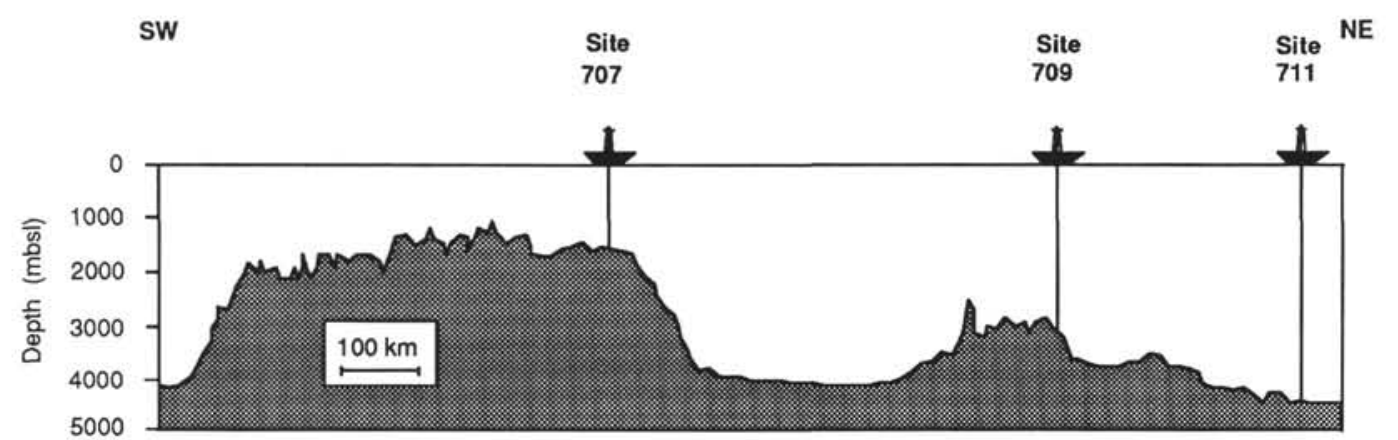

Site 707 (1541.4 mbsl)

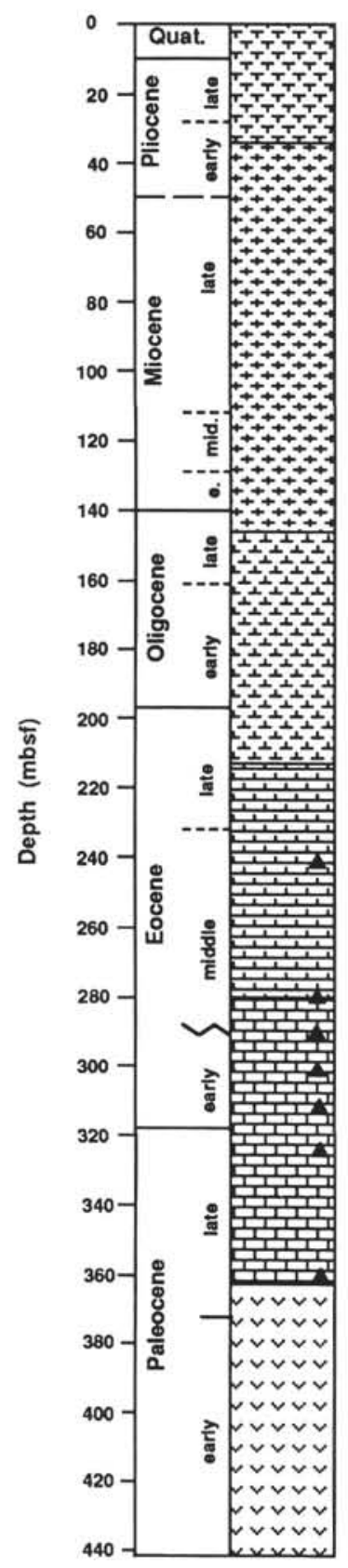

Site 711 (4428.2 mbsl) (3038.2 mbsl)

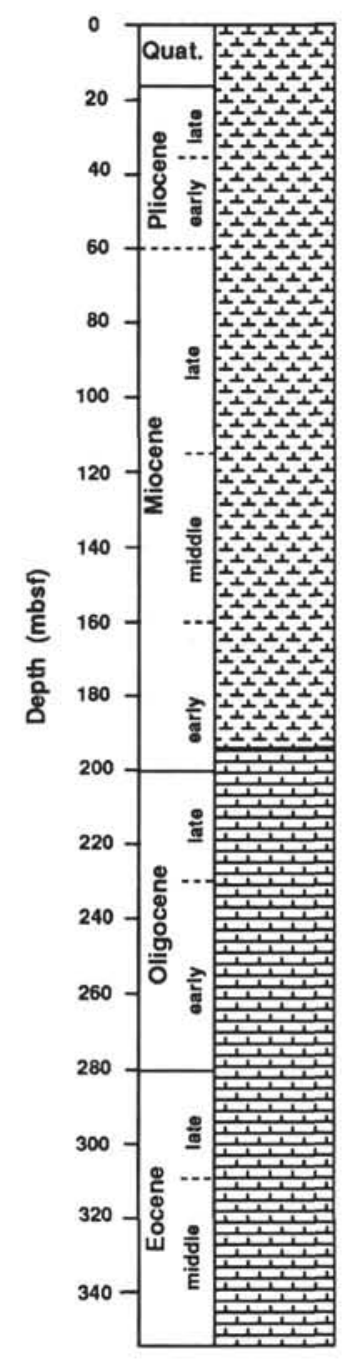

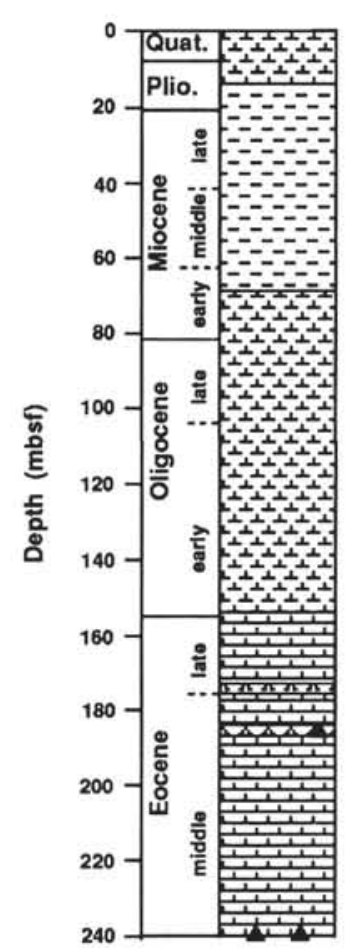

Figure 2. Record of a bathymetric profile over the Mascarene Plateau in the southwest and Madingley Rise in the northeast obtained during Leg 115 (Backman, Duncan, et al., 1988) and positions of the sites studied. General lithologic columns and ages of Sites 707,709 , and 711 are recorded. 


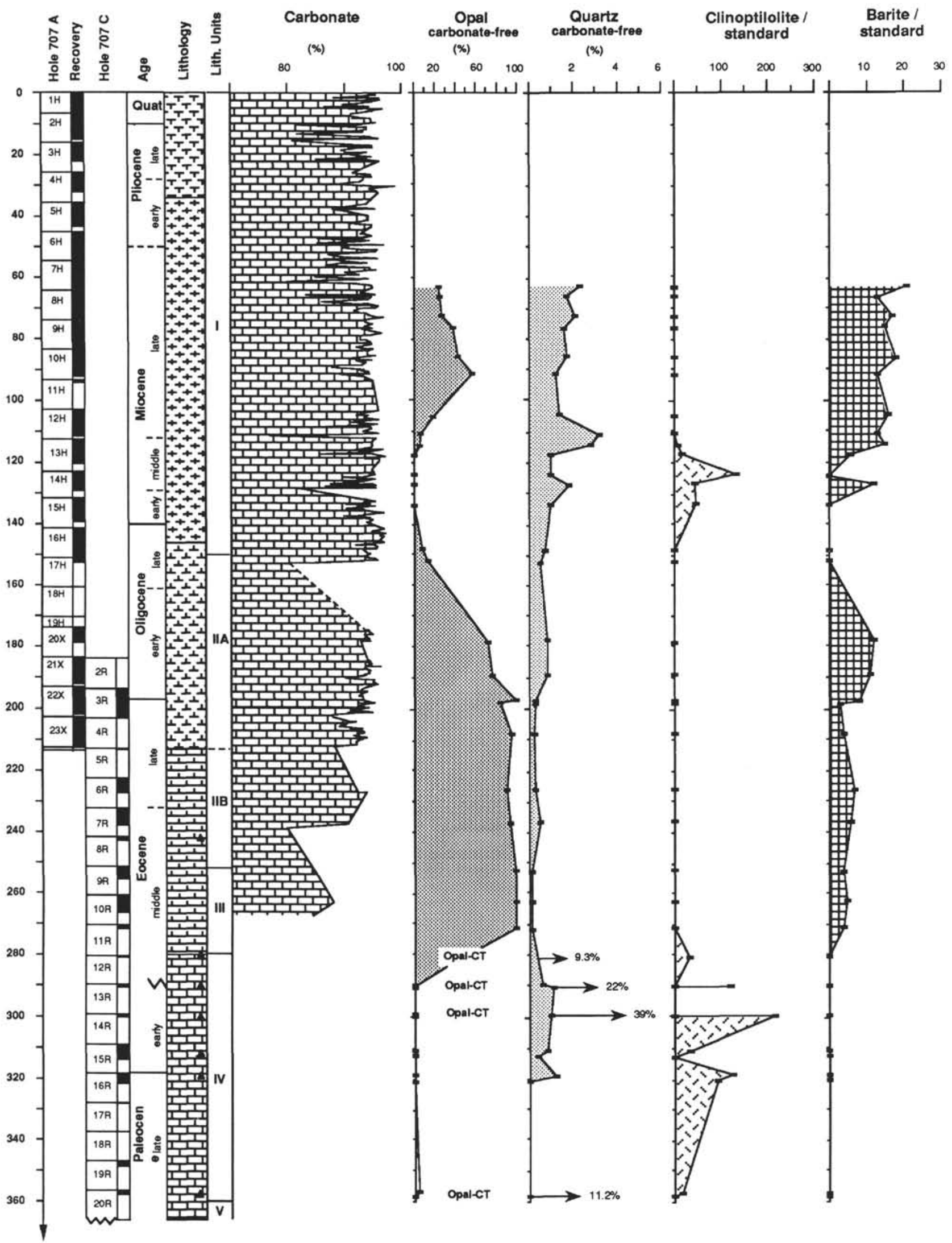

Figure 3. Lithotypes, ages, carbonate contents (from Backman, Duncan, et al., 1988), and results from XRD measurements at Site 707. Quantitative patterns of the mineralogical data were calculated on a carbonate-free basis. Opal-A and quartz contents were calculated by means of calibration curves. The clinoptilolite pattern was determined from the ratio $I_{(020) \text { clinoptilolite }}$ to $I_{(012) \text { corundum }} \times 100$. Barite was calculated from the ratio of $\mathrm{I}_{(121) \text { barite }}$ to $\mathrm{I}_{(012) \text { corundum }} \times 100$. 


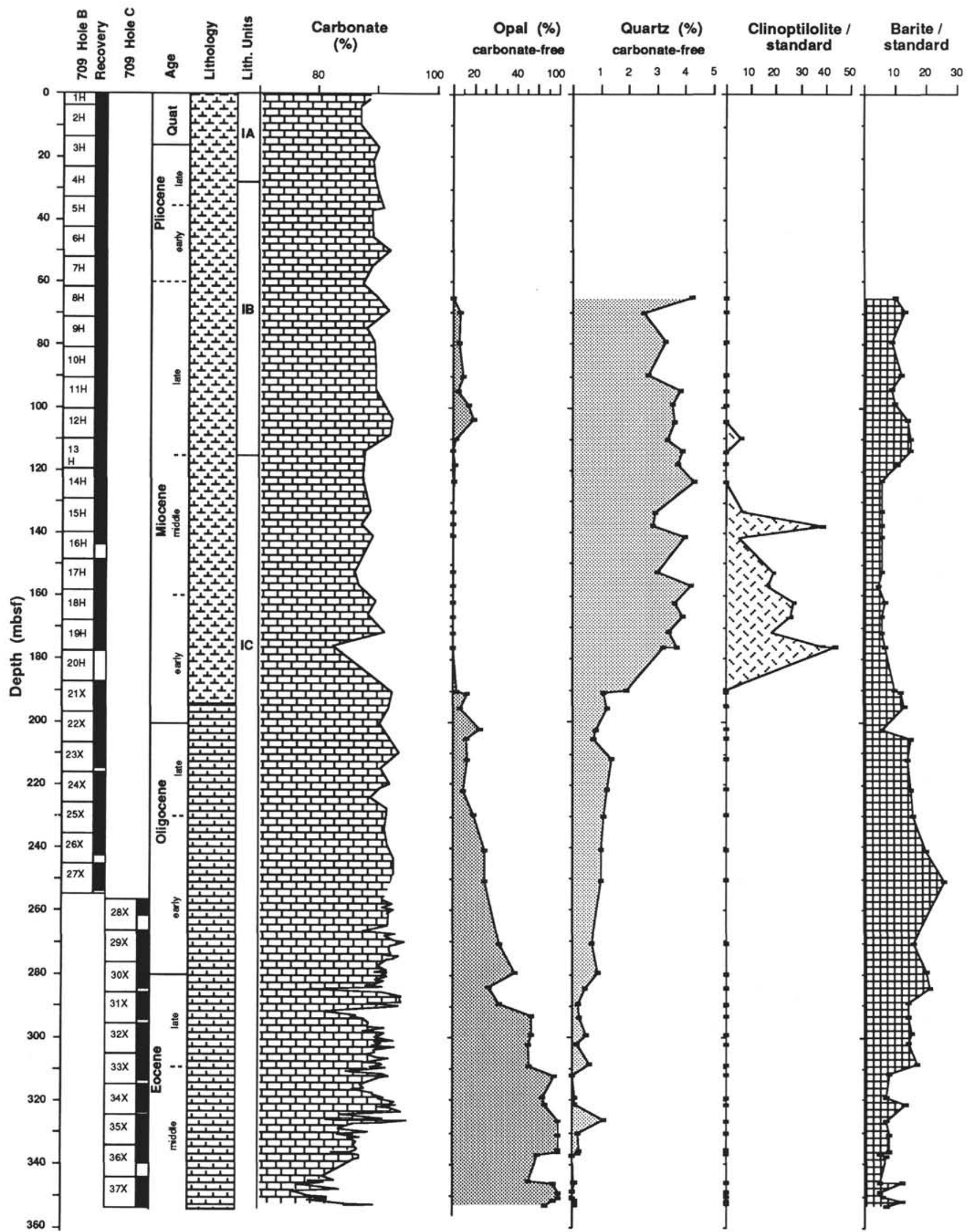

Figure 4. Lithotypes, ages, carbonate contents (from Backman, Duncan, et al., 1988), and results from XRD measurements at Site 709. Quantitative patterns of the mineralogical data were calculated on a carbonate-free basis. Opal-A and quartz contents were calculated by means of calibration curves. The clinoptilolite pattern was determined from the ratio $I_{(020) \text { clinoptilolite }}$ to $I_{(012) \text { corundum }} \times 100$. Barite was calculated from the ratio of $\mathrm{I}_{(121) \text { barite }}$ to $\mathrm{I}_{(012) \text { corundum }} \times 100$. 


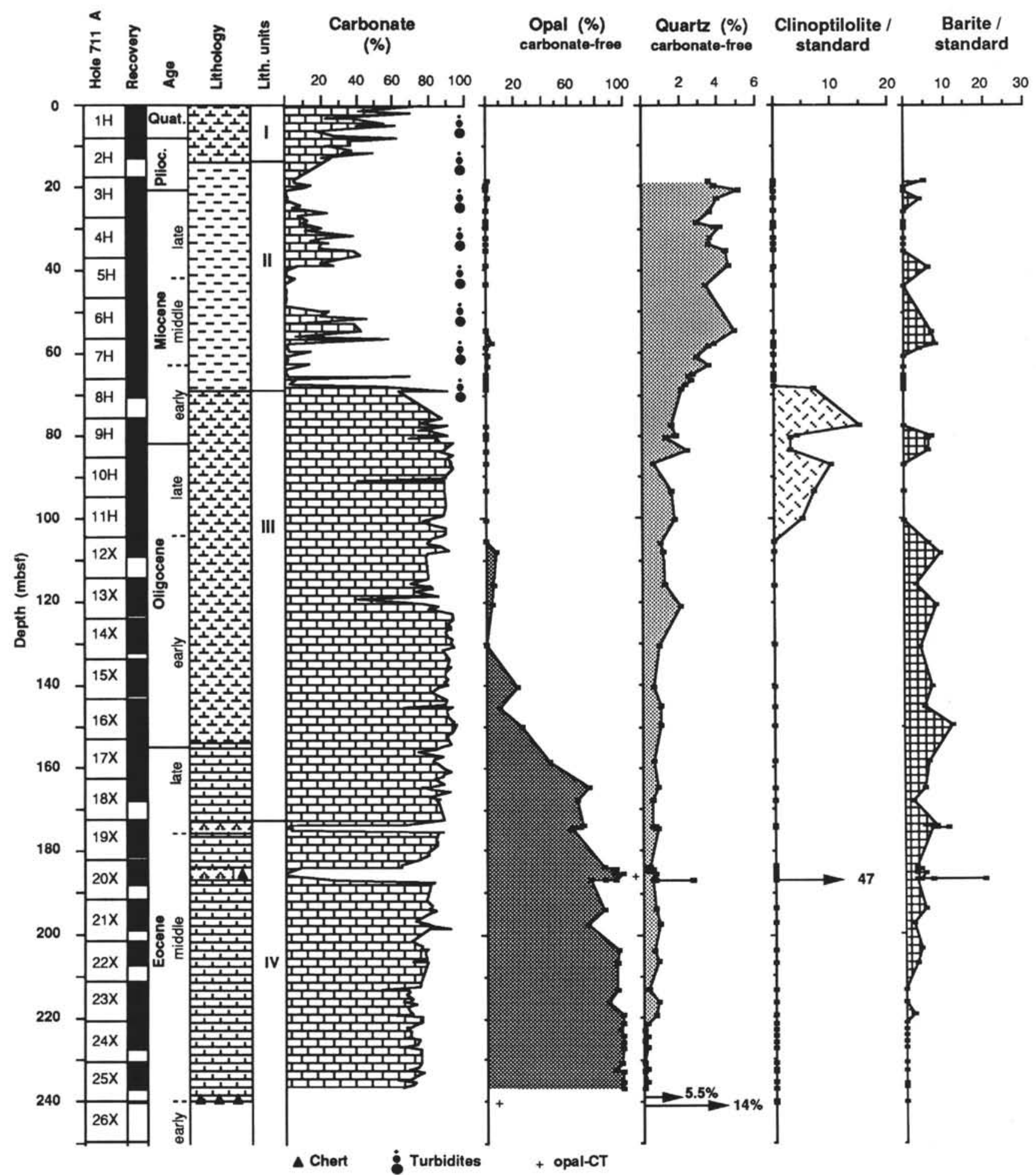

Figure 5. Lithotypes, ages, carbonate contents (from Backman, Duncan, et al., 1988) and results from XRD measurements at Site 711. Quantitative patterns of the mineralogical data were calculated on a carbonate-free basis. Opal-A and quartz contents were calculated by means of calibration curves. The clinoptilolite pattern was determined from the ratio $I_{(020) \text { clinoptilolite }}$ to $I_{(012) \text { corundum }} \times 100$. Barite was calculated from the ratio of $I_{(121) \text { barite }}$ to $I_{(012) \text { corundum }} \times 100$. 
present. The results of our quantitative analyses are presented in Figures 3, 4, and 5 and are listed in Tables 1-3.

\section{Quartz}

Contents of detrital quartz in the diagenetically less affected intervals vary between $0 \%$ and $5 \%$ of the carbonate-free fraction. The highest contents are reached at northernmost Site 711 (Fig. 5), whereas the sediments at southernmost Site 707 (Fig. 3) show the lowest contents; Site 709 (Fig. 4) contents range between these two. A major trend from generally lower quartz amounts in Oligocene and Eocene sediments to higher quartz contents in Miocene sediments is documented in all three sites (Figs. 3-5). This trend is best seen in the record of Site 709, in which quartz amounts reach minimum values below $0.2 \%$ downhole during the late and middle Eocene, where maximum values of biogenic opal were recorded. In the early Miocene a sharp step occurs, with values ranging from $1 \%$ to around $3 \%$ and $4 \%$. In early Eocene sediments, which were only recovered at Site 707, a slight increase in quartz content can be correlated with the occurrence of chert. Quartz contents measured from chert samples vary between $10 \%$ and $40 \%$.

\section{Biogenic Opal}

In general, the pattern of opal-A abundances correlates well among all three sites. The XRD records in early Eocene and $\mathrm{Pa}$ leocene sediments, only recovered at Site 707, do not show any evidence of biogenic opal. However, some cherty intervals exist within this sequence; they are predominantly composed of opalCT $(50 \%-80 \%)$ and, to a lesser extent, of quartz and carbonate (Fig. 3).

Maximum opal-A contents up to $99 \%$ of the carbonate-free basis occur during the Eocene, decrease upsection, and reach minimum values in Miocene sediments. This upward decrease in biogenic opal at all sites corresponds well with a general increase in the carbonate record, changing from average values of $80 \%$ in Eocene sediments to values around $90 \%$ in Oligocene deposits (Figs. 3-5). With regard to these similarities in the opal-A records, we also observe regional differences from north to south. A pattern of latitudinal opal-A distribution is documented in the late Eocene, with high values at southernmost Site 707 and low values at northernmost Site 711 (Figs. 3-5). A more or less constant high level (up to $99 \%$ ) was reached

Table 1. Results from X-ray diffraction analyses (taken from the carbonate-free sample) from sediments at Site 707 .

\begin{tabular}{|c|c|c|c|c|c|c|c|c|}
\hline $\begin{array}{l}\text { Core, section } \\
\text { interval }(\mathrm{cm})\end{array}$ & $\begin{array}{l}\text { Depth } \\
\text { (mbsf) }\end{array}$ & $\begin{array}{c}\text { Quartz } \\
(\%)\end{array}$ & $\mathrm{Cl} / \mathrm{Co}$ & $\mathrm{Ba} / \mathrm{Co}$ & $\mathrm{CT} / \mathrm{Co}$ & $\begin{array}{c}\mathrm{CaCO}_{3} \\
(\%)\end{array}$ & $\begin{array}{l}\text { Opal } \\
(\%)\end{array}$ & Comments \\
\hline \multicolumn{9}{|l|}{$115-707 \mathrm{~A}-$} \\
\hline $7 \mathrm{H}-6,90$ & 63.10 & 2.3 & 0 & 21 & & 94.95 & 23.7 & \\
\hline $8 \mathrm{H}-2,54$ & 66.34 & 1.7 & 0 & 13 & & 94.70 & 25.4 & \\
\hline $8 \mathrm{H}-6,56$ & 72.36 & 2.1 & 0 & 17 & & 94.08 & 27.2 & \\
\hline $9 \mathrm{H}-2,84$ & 76.34 & 1.6 & 0 & 15 & & 94.98 & 38.2 & \\
\hline $10 \mathrm{H}-2,83$ & 85.93 & 1.7 & 0 & 18 & & 90.43 & 42.0 & \\
\hline $10 \mathrm{H}-6,45$ & 91.55 & 1.2 & 0 & 13 & & 89.16 & 55.6 & \\
\hline $12 \mathrm{H}-2,53$ & 104.83 & 1.4 & 0 & 16 & & 94.08 & 18.7 & \\
\hline $12 \mathrm{H}-6,50$ & 110.80 & 3.2 & 0 & 13 & & 86.11 & 6.6 & \\
\hline $13 \mathrm{H}-2,48$ & 114.38 & 2.8 & 7 & 15 & & 94.68 & 5.4 & \\
\hline $13 \mathrm{H}-4,52$ & 117.42 & 1.0 & 18 & 8 & & 95.00 & 0.0 & \\
\hline $14 \mathrm{H}-2,54$ & 124.04 & 1.0 & 133 & 0 & & 93.48 & 0.0 & \\
\hline $14 \mathrm{H}-4,47$ & 128.97 & 1.8 & 44 & 12 & & 95.55 & 0.0 & \\
\hline $15 \mathrm{H}-2,58$ & 133.88 & 1.0 & 47 & 0 & & 92.19 & 0.4 & \\
\hline $18 \mathrm{H}-5,103$ & 148.33 & 0.7 & 0 & 0 & & 93.58 & 8.5 & \\
\hline $17 \mathrm{H}-1,125$ & 152.25 & 0.5 & 0 & 0 & & 84.15 & 13.3 & \\
\hline $20 X-3,143$ & 178.13 & 0.8 & 0 & 12 & & 92.80 & 71.7 & \\
\hline $21 X-4,141$ & 189.31 & 0.8 & 0 & 11 & & 93.68 & 78.2 & \\
\hline $22 \times-3,140$ & 197.50 & 0.3 & 0 & 8 & & 92.22 & 99.0 & \\
\hline $23 X-4,85$ & 208.05 & 0.2 & 0 & 4 & & 91.38 & 94.8 & \\
\hline \multicolumn{9}{|l|}{$115-707 \mathrm{C}$ - } \\
\hline $3 \mathrm{R}-4,23$ & 198.23 & 0.3 & 0 & 3 & & 92.03 & 83.9 & \\
\hline $6 \mathrm{R}-3,65$ & 226.20 & 0.3 & 0 & 7 & & 92.26 & 90.0 & \\
\hline $8 \mathrm{R}-3,85$ & 228.20 & 0.3 & 0 & 7 & & 92.28 & 90.0 & \\
\hline $7 \mathrm{R}-3,142$ & 238.52 & 0.5 & 0 & 8 & & 90.54 & 94.2 & \\
\hline $9 \mathrm{R}-1,128$ & 252.88 & 0.1 & 0 & 4 & & 84.90 & 99.0 & \\
\hline $10 \mathrm{R}-2,33$ & 282.83 & 0.1 & 0 & 5 & & 87.83 & 99.0 & \\
\hline $11 \mathrm{R}-1,85$ & 271.45 & 0.1 & 0 & 4 & & 1.92 & 99.0 & \\
\hline $12 \mathrm{R}-1,42$ & 280.72 & 9.3 & 33 & 0 & 60 & & & Chert \\
\hline $13 \mathrm{R}-1,30$ & 290.10 & 0.8 & 0 & 0 & & 0.87 & & \\
\hline $13 \mathrm{R}-1,53$ & 290.33 & 1.1 & 120 & 0 & & & 0.0 & \\
\hline $13 \mathrm{R}-1,87$ & 290.47 & 22.0 & 0 & 0 & 149 & & 0.0 & Chert \\
\hline $14 \mathrm{R}-1,15$ & 299.45 & 39.0 & 0 & 0 & 37 & & 0.0 & Chert \\
\hline $14 \mathrm{R}-1,34$ & 299.84 & 1.0 & 217 & 0 & & 0.27 & & \\
\hline $15 R-2,50$ & 310.90 & 0.8 & 34 & 0 & & & 0.0 & Glauconitic limestone \\
\hline $15 \mathrm{R}-3,80$ & 312.50 & 0.4 & 0 & 0 & & 0.48 & & Glauconitic limestone \\
\hline $18 \mathrm{R}-1,85$ & 318.85 & 1.2 & 125 & 0 & & 0.33 & & \\
\hline $18 \mathrm{R}-2,100$ & 320.70 & 0.0 & 94 & 0 & & & 0.0 & \\
\hline $20 \mathrm{R}-1,41$ & 357.11 & 8.0 & 18 & 0 & 31 & 0.20 & & Partly chert \\
\hline 20R-CC, 11 & 358.41 & 11.2 & 0 & 0 & & & 0.0 & Outside of concretion \\
\hline 20R-CC, 12 & 358.42 & 0.0 & 0 & 0 & & & 0.0 & Center of concretion \\
\hline
\end{tabular}

Note: $\mathrm{Cl} / \mathrm{Co}=$ ratio of clinoptilolite to the corundum standard, $\mathrm{Ba} / \mathrm{Co}=$ ratio of barium to the corundum standard, and $\mathrm{CT} / \mathrm{CO}=$ ratio of opal-CT to the corundum standard. 
Table 2. Results from X-ray diffraction analyses (taken from the carbonate-free sample) from sediments at Site 709.

\begin{tabular}{|c|c|c|c|c|c|c|}
\hline $\begin{array}{l}\text { Core, section } \\
\text { interval }(\mathrm{cm})\end{array}$ & $\begin{array}{l}\text { Depth } \\
\text { (mbsf) }\end{array}$ & $\begin{array}{c}\text { Quartz } \\
(\%)\end{array}$ & $\mathrm{Cl} / \mathrm{Co}$ & $\mathrm{Ba} / \mathrm{Co}$ & $\begin{array}{c}\mathrm{CaCO}_{3} \\
(\%)\end{array}$ & $\begin{array}{l}\text { Opal } \\
(\%)\end{array}$ \\
\hline 115-709B-8H-3, 56 & 65.25 & 4.22 & 0 & 10 & 90.15 & 0 \\
\hline 115-709B-8H-6, 62 & 69.80 & 2.52 & 0 & 13 & 96.96 & 7 \\
\hline 115-709B-9H-6, 62 & 79.40 & 3.31 & 0 & 9 & 89.54 & 8 \\
\hline 115-709B-10H-6, 143 & 89.92 & 2.67 & 0 & 12 & 92.50 & 10 \\
\hline $115-709 \mathrm{~B}-11 \mathrm{H}-3,86$ & 94.45 & 3.81 & 0 & 9 & 89.84 & 5 \\
\hline 115-709B-11H-6, 79 & 98.89 & 3.54 & 0 & 10 & 91.16 & 15 \\
\hline I15-709B-12H-3, 83 & 104.13 & 3.60 & 0 & 14 & 92.66 & 19 \\
\hline $115-709 \mathrm{~B}-12 \mathrm{H}-6,70$ & 110.00 & 3.36 & 6 & 15 & & 3 \\
\hline $115-709 \mathrm{R}-13 \mathrm{H}-3,90$ & 113.80 & 3.90 & 0 & 15 & 88.14 & 0 \\
\hline $115-709 \mathrm{~B}-13 \mathrm{H}-6,66$ & 118.05 & 3.78 & 0 & 11 & 87.54 & 2 \\
\hline $115-709 \mathrm{~B}-14 \mathrm{H}-3,84$ & 123.34 & 4.30 & 0 & 6 & 85.81 & 1 \\
\hline $115-709 \mathrm{~B}-15 \mathrm{H}-3,89$ & 133.09 & 2.92 & 7 & 6 & 88.87 & 0 \\
\hline 115-709B-15H-6, 71 & 137.41 & 2.85 & 39 & 6 & 87.44 & 0 \\
\hline $115-709 \mathrm{~B}-16 \mathrm{H}-2,84$ & 141.24 & 4.00 & 5 & 6 & 89.44 & 0 \\
\hline $115-709 \mathrm{~B}-17 \mathrm{H}-3,85$ & 152.45 & 3.00 & 19 & 6 & 86.26 & 0 \\
\hline 115-709B-17H-6, 59 & 156.69 & 4.17 & 17 & 5 & 87.06 & 0 \\
\hline 115-709B-18H-3, 81 & 162.11 & 3.60 & 27 & 7 & 89.90 & 0 \\
\hline $115-709 \mathrm{~B}-18 \mathrm{H}-6,64$ & 166.44 & 3.90 & 26 & 6 & 88.49 & 0 \\
\hline 115-709B-19H-3, 77 & 171.77 & 3.40 & 18 & 6 & 91.41 & 0 \\
\hline 115-709B-19H-6, 71 & 176.21 & 3.70 & 39 & 7 & 82.57 & 0 \\
\hline $115-709 \mathrm{C}-19 \mathrm{H}-7,28$ & 176.28 & 3.22 & 44 & 7 & & 0 \\
\hline 115-709B-21X-1, 75 & 189.85 & 1.90 & 0 & 10 & & 4 \\
\hline $115-709 \mathrm{~B}-21 \mathrm{X}-2,51$ & 190.71 & 1.11 & 0 & 12 & & 14 \\
\hline $115-709 \mathrm{~B}-21 \mathrm{X}-6,48$ & 195.18 & 1.21 & 0 & 13 & 92.04 & 7 \\
\hline $115-709 \mathrm{~B}-22 \mathrm{X}-3,75$ & 202.45 & 0.80 & 0 & 6 & 90.50 & 25 \\
\hline 115-709B-22X-6, 122 & 205.52 & 0.75 & 0 & 15 & 93.14 & 13 \\
\hline $115-709 \mathrm{~B}-23 \mathrm{X}-3,56$ & 211.96 & 1.40 & 0 & 14 & 93.77 & 14 \\
\hline 115-709B-24X-3, 57 & 221.57 & 1.20 & 0 & 15 & 92.19 & 10 \\
\hline 115-709B-25X-2, 57 & 229.77 & 1.10 & 0 & 16 & 91.79 & 19 \\
\hline $115-709 \mathrm{~B}-26 \mathrm{X}-3,57$ & 240.97 & 1.00 & 0 & 20 & 91.76 & 30 \\
\hline 115-709B-27X-3, 67 & 250.67 & 1.00 & 0 & 26 & 92.95 & 30 \\
\hline $115-709 C-29 X-3,65$ & 270.05 & 0.70 & 0 & 16 & 94.59 & 44 \\
\hline $115-709 C-30 X-3,60$ & 279.70 & 0.90 & 0 & 20 & 91.75 & 58 \\
\hline $115-709 C-30 X-6,62$ & 284.22 & 0.43 & 0 & 21 & 90.97 & 34 \\
\hline $115-709 \mathrm{C}-31 \mathrm{X}-3,52$ & 289.32 & 0.21 & 0 & 14 & 88.72 & 44 \\
\hline $115-709 C-31 X-6,108$ & 293.38 & 0.25 & 0 & 14 & 87.75 & 74 \\
\hline $115-709 C-32 X-3,58$ & 298.98 & 0.49 & 0 & 15 & 91.30 & 74 \\
\hline $115-709 C-32 X-5,59$ & 301.99 & 0.18 & 0 & 14 & 90.74 & 71 \\
\hline $115-709 C-38 X-8,68$ & 308.78 & 0.59 & 0 & 17 & 91.00 & 72 \\
\hline $115-709 C-83 X-5,65$ & 311.75 & 0.00 & 0 & 8 & 90.92 & 95 \\
\hline $115-709 C-34 X-8,127$ & 319.07 & 0.10 & 0 & 7 & 91.08 & 84 \\
\hline $115-709 C-34 X-5,66$ & 321.46 & 0.10 & 0 & 13 & 98.28 & 86 \\
\hline $115-709 C-85 X-2,54$ & 326.44 & 1.10 & 0 & 7 & 81.51 & 99 \\
\hline $115-709 C-35 X-5,80$ & 330.70 & 0.20 & 0 & 8 & 86.11 & 99 \\
\hline $115-709 C-36 X-2,80$ & 335.90 & 0.25 & 0 & 8 & & 99 \\
\hline $115-709 C-86 X-2,92$ & 336.52 & 0.20 & 0 & 5 & 82.46 & 99 \\
\hline $115-709 C-86 X-8,80$ & 337.40 & 0.00 & 0 & 7 & & 80 \\
\hline $115-709 \mathrm{C}-87 \mathrm{X}-2,80$ & 345.70 & 0.05 & 0 & 5 & & 71 \\
\hline $115-709 C-87 X-2,71$ & 346.11 & 0.10 & 0 & 12 & 81.16 & 94 \\
\hline $115-709 C-87 X-4,50$ & 348.90 & 0.00 & 0 & 5 & & 99 \\
\hline $115-709 C-37 X-5,68$ & 350.58 & 0.00 & 0 & 6 & 81.68 & 99 \\
\hline $115-709 C-87 X-6,80$ & 351.70 & 0.10 & 0 & 12 & & 94 \\
\hline $115-709 \mathrm{C}-87 \mathrm{X}-7,2$ & 352.91 & 0.10 & 0 & 7 & 89.49 & 86 \\
\hline
\end{tabular}

Note: $\mathrm{Cl} / \mathrm{Co}=$ ratio of clinoptilolite to the corundum standard, and $\mathrm{Ba} / \mathrm{Co}=\mathrm{ra}$ tio of barium to the corundum standard.

for the middle Eocene. Similar trends from low opal-A contents at Site $711(0 \%-25 \%$; Fig. 5), intermediate values at Site 709 $(10 \%-57 \%$, Fig. 4), and higher contents at Site $707(8 \%-99 \%$, Fig. 3) appear in the Oligocene.

At Site 711 biogenic opal is almost absent from the late Oligocene to the late Miocene. This fits well with the microscopic observations of Backman, Duncan, et al. (1988), which is that Cores $115-711 \mathrm{~A}-2 \mathrm{H}$ through $-12 \mathrm{X}$ are barren of radiolarians. In contrast, XRD records from Sites 707 and 709 show opal-A in early and late Oligocene and late Miocene sediments, although no opal-A was recorded in the early and middle Miocene. The same pattern of latitudinal opal-A distribution is documented for the late Miocene, with opal-A contents ranging between $5 \%$ and $19 \%$ at Site 709 and exceeded by southernmost Site 707 (Fig. 3), with average values of $\sim 30 \%$ and maxima at $56 \%$.
The biogenic silica composition is dominated by radiolarians and, to a lesser extent, by sponge spicules, diatoms, and silicoflagellates. The preservation of biogenic opaline particles varies extremely. Given our SEM observations, strong opal corrosion can be observed in sediments that contain low amounts of opalA. Most siliceous particles are partly fragmented (Plate 2, Figs. 1 and 4). Two modes of dissolution structures of opaline skeletons were distinguished under the SEM. In late Oligocene sediments from Site 707, where biogenic opal reaches up to $10 \%$ of the carbonate-free fraction (Fig. 3), we observed very rough surfaces and numerous corrosion scars (Plate 2, Fig. 4) or single pits (Plate 2, Fig. 2). Such surfaces are the result of opal dissolution exhibiting the very fine globose structures of microspheres, which are ultrastructural elements of the opaline skeletons (Plate 2, Figs. 3 and 5). 
Table 3. Results from $\mathbf{X}$-ray diffraction analyses (taken from the carbonate-free sample) from sediments at Site 711 .

\begin{tabular}{|c|c|c|c|c|c|c|c|}
\hline $\begin{array}{l}\text { Core, section } \\
\text { interval }(\mathrm{cm})\end{array}$ & $\begin{array}{l}\text { Depth } \\
\text { (mbsf) }\end{array}$ & $\begin{array}{c}\text { Quartz } \\
(\%)\end{array}$ & $\mathrm{Cl} / \mathrm{Co}$ & $\mathrm{Ba} / \mathrm{Co}$ & $\begin{array}{c}\mathrm{CaCO}_{3} \\
(\%)\end{array}$ & $\begin{array}{l}\text { Opal } \\
(\%)\end{array}$ & Comments \\
\hline \multicolumn{8}{|l|}{$115-711 \mathrm{~A}-$} \\
\hline $3 \mathrm{H}-1,76$ & 18.46 & 3.5 & 0 & 5 & & 1 & Feldspar \\
\hline $3 \mathrm{H}-2,62$ & 19.82 & 8.8 & 0 & 0 & 10.1 & 0 & Feldspar \\
\hline $3 \mathrm{H}-3,2 \mathrm{I}$ & 20.91 & 5.1 & 0 & 0 & 0.8 & 0 & Feldspar \\
\hline $3 \mathrm{H}-4,62$ & 22.82 & 4.0 & 0 & 4 & 1.0 & 1 & Feldspar \\
\hline $3 \mathrm{H}-6,62$ & 25.82 & 8.6 & 0 & 0 & 28.5 & 0 & Feldspar \\
\hline $4 \mathrm{H}-1,123$ & 28.58 & 2.9 & 0 & 0 & & 0 & Feldspar \\
\hline $4 \mathrm{H}-2,62$ & 29.42 & 3.8 & 0 & 0 & 20.5 & 0 & Feldspar \\
\hline $4 \mathrm{H}-2,105$ & 29.85 & 4.1 & 0 & 0 & & 0 & Feldspar \\
\hline $4 \mathrm{H}-4,62$ & 82.42 & 8.6 & 0 & 0 & 14.2 & 0 & Feldspar \\
\hline $4 \mathrm{H}-5,52$ & 38.82 & 3.5 & 0 & 0 & & 0 & Feldspar \\
\hline $4 \mathrm{H}-6,62$ & 35.42 & 4.4 & 0 & 0 & & 0 & Feldspar \\
\hline $5 \mathrm{H}-2,62$ & 39.02 & 4.6 & 0 & 6 & 6.6 & 0 & Feldspar \\
\hline $5 \mathrm{H}-5,62$ & 43.52 & 3.4 & 0 & 0 & & 0 & Feldspar \\
\hline $6 \mathrm{H}-6,62$ & 54.72 & 4.9 & 0 & 7 & 42.2 & 0 & Feldspar \\
\hline $7 \mathrm{H}-1,127$ & 57.57 & 3.8 & 0 & 8 & & 4 & Feldspar \\
\hline $7 \mathrm{H}-2,62$ & 58.42 & 3.5 & 0 & 4 & 0.6 & 0 & Feldspar \\
\hline $7 \mathrm{H}-3,127$ & 60.57 & 2.9 & 0 & 0 & 14.1 & 1 & Feldspar \\
\hline $7 \mathrm{H}-5,78$ & 63.08 & 3.5 & 0 & 0 & & 1 & Feldspar \\
\hline $7 \mathrm{H}-6,127$ & 65.07 & 2.7 & 0 & 0 & & 0 & Feldspar \\
\hline $7 \mathrm{H}-7,20$ & 65.50 & 2.5 & 0 & 0 & & 0 & Feldspar \\
\hline $8 \mathrm{H}-1,42$ & 66.48 & 2.6 & 0 & 0 & & 0 & Feldspar \\
\hline $8 \mathrm{H}-2,18$ & 67.68 & 2.3 & 0 & 0 & & 0 & Feldspar \\
\hline $8 \mathrm{H}-2,94$ & 68.44 & 2.1 & 7 & 0 & & 0 & Feldspar \\
\hline $9 \mathrm{H}-2,59$ & 77.69 & 1.6 & 15 & 0 & 90.0 & 0 & \\
\hline $9 \mathrm{H}-3,14$ & 80.04 & 1.8 & 4 & 7 & & 0 & \\
\hline $9 \mathrm{H}-4,55$ & 80.65 & 1.3 & 3 & 6 & 84.0 & 0 & \\
\hline $9 \mathrm{H}-6,50$ & 83.60 & 2.4 & 3 & 6 & 83.5 & 0 & \\
\hline $10 \mathrm{H}-2,13$ & 86.86 & 0.6 & 10 & 0 & & 0 & \\
\hline $10 \mathrm{H}-6,97$ & 93.67 & 1.6 & 7 & 0 & 88.0 & 0 & \\
\hline $11 \mathrm{H}-4,102$ & 100.32 & 1.7 & 5 & 0 & 76.2 & 0 & \\
\hline $12 \mathrm{H}-1,108$ & 105.80 & 1.0 & 0 & 6 & 78.4 & 0 & \\
\hline $12 \mathrm{H}-3,82$ & 108.32 & 1.1 & 0 & 9 & 79.1 & 7 & \\
\hline $13 \mathrm{H}-2,35$ & 116.05 & 1.2 & 0 & 3 & 80.0 & 5 & \\
\hline $13 \mathrm{H}-5,72$ & 120.92 & 2.0 & 0 & 8 & 84.3 & 4 & \\
\hline $14 \mathrm{H}-5,57$ & 130.47 & 0.9 & 0 & 4 & & 0 & \\
\hline $15 \mathrm{H}-5,77$ & 140.37 & 0.6 & 0 & 7 & 84.2 & 22 & \\
\hline $16 \mathrm{H}-2,46$ & 145.26 & 1.0 & 0 & 5 & & 10 & \\
\hline $16 \mathrm{H}-5,48$ & 149.78 & 1.0 & 0 & 12 & 92.0 & 25 & \\
\hline $17 \mathrm{H}-5,66$ & 158.66 & 0.6 & 0 & 6 & & 46 & \\
\hline $18 \mathrm{H}-2,62$ & 164.82 & 0.8 & 0 & 5 & 75.3 & 75 & \\
\hline $18 \mathrm{H}-4,62$ & 167.82 & 0.5 & 0 & 2 & 82.8 & 66 & \\
\hline $19 X-1,146$ & 173.86 & 0.5 & 0 & 8 & & 70 & \\
\hline $19 \times-2,9$ & 173.99 & 0.5 & 0 & 7 & & 65 & \\
\hline $19 \times-2,15$ & 174.05 & 0.6 & 0 & 5 & 2.6 & 65 & \\
\hline $19 \times-2,44$ & 174.34 & 0.6 & 0 & 8 & & 63 & \\
\hline $19 \times-2,47$ & 174.37 & 0.7 & 0 & 11 & 3.2 & 68 & \\
\hline $19 X-2,75$ & 174.65 & 0.7 & 0 & 8 & & 61 & \\
\hline $19 \times-2,79$ & 174.69 & 0.8 & 0 & 7 & & 63 & \\
\hline $20 X-2,18$ & 183.78 & 0.4 & 0 & 3 & & 85 & \\
\hline $20 X-2,61$ & 184.21 & 0.3 & 0 & 4 & 7.8 & 92 & \\
\hline $20 X-2,84$ & 184.44 & 0.5 & 0 & 3 & & 94 & \\
\hline $20 \mathrm{X}-3,13$ & 185.23 & 0.3 & 0 & 3 & & 93 & \\
\hline $20 X-3,43$ & 185.53 & 0.7 & 0 & 5 & & 99 & \\
\hline $20 X-4,23$ & 186.83 & 0.6 & 0 & 4 & 26.8 & 94 & \\
\hline $20 X-4,28$ & 186.89 & 0.7 & 0 & 7 & & 86 & \\
\hline $20 \times-4,31$ & 186.91 & 2.6 & 47 & 20 & & 1 & Opal-CT $/ \mathrm{Co}=63$ \\
\hline $20 \times-4,48$ & 187.08 & 0.5 & 0 & 3 & & 76 & \\
\hline $21 \times-2,62$ & 193.82 & 0.7 & 0 & 5 & 79.1 & 85 & \\
\hline $21 \times-5,62$ & 197.32 & 0.9 & 0 & 2 & & 73 & \\
\hline $22 X-2,62$ & 203.52 & 0.6 & 0 & 4 & & 96 & \\
\hline $22 \times-4,62$ & 206.52 & 0.8 & 0 & 3 & & 95 & \\
\hline $23 X-2,62$ & 213.22 & 0.3 & 0 & 0 & & 95 & \\
\hline $23 \times-4,62$ & 216.22 & 0.8 & 0 & 0 & 64.3 & 88 & \\
\hline $23 \times-6,53$ & 219.13 & 0.7 & 0 & 2 & & 99 & \\
\hline $24 X-1,62$ & 221.32 & 0.2 & 0 & 0 & 71.5 & 99 & \\
\hline $24 X-2,61$ & 222.81 & 0.1 & 0 & 0 & & 97 & \\
\hline $24 X-3,62$ & 224.32 & 0.2 & 0 & 0 & 69.0 & 99 & \\
\hline $24 X-4,61$ & 225.81 & 0.1 & 0 & 0 & & 99 & \\
\hline $24 X-5,62$ & 227.32 & 0.2 & 0 & 0 & 65.6 & 99 & \\
\hline $25 X-1,52$ & 230.82 & 0.1 & 0 & 0 & & 98 & \\
\hline $25 X-2,62$ & 232.42 & 0.2 & 0 & 0 & 67.7 & 94 & \\
\hline $25 X-3,52$ & 232.82 & 0.1 & 0 & 0 & & 99 & \\
\hline $25 \times-4,62$ & 235.42 & 0.2 & 0 & 0 & 71.6 & 99 & \\
\hline $25 \times-5,52$ & 236.82 & 0.1 & 0 & 0 & & 99 & \\
\hline $26 \mathrm{X}-\mathrm{CC}, 5$ & 240.05 & 5.5 & 0 & 0 & 16.0 & 0 & Opal-CT $/ \mathrm{Co}=56$ \\
\hline $26 \mathrm{X} \cdot \mathrm{CC}, 30$ & 240.30 & 14.0 & 0 & 0 & 11.0 & 0 & Opal-CT $/ \mathrm{Co}=41$ \\
\hline
\end{tabular}

Note: $\mathrm{Cl} / \mathrm{Co}=$ ratio of clinoptilolite to the corundum standard, and $\mathrm{Ba} / \mathrm{Co}=$ ratio of barium to the corundum standard. 
Completely different dissolution structures are revealed in the lower and middle Miocene section at Site 707 and to a very small extent at Site 709. Although only traces of biogenic opalA are apparent within this interval (Figs. 3 and 4), numerous sponge spicules are found in the carbonate-free $>40-\mu \mathrm{m}$ fraction (Plate 1, Fig. 1). The spicules there do not show the typical spongy surface structure. The skeletons contain smooth but scarred surfaces (Plate 1, Figs. 2, 3, and 4) and are provided with numerous corrosion pits (Plate 1). The pits are characterized by conspicuous circular, well- rounded holes that reveal sharp edges (Plate 1, Fig. 3) and that are often observed going so deep that they reach the axial channel. Such circular, rounded corrosion structures occur as single pits or holes as well as groups of corrosion pits (Plate 1, Fig. 4). Because of several generations of corrosion, strongly altered sponge spicules appear with different sizes and penetrations of the circular corrosion pits (Plate 1, Fig. 5). Smooth parallel ribs and grooves are visible at the inner wall of the corrosive deepening, which most probably represent details of the concentric laminated inner structure of the opaline skeletal material (Plate 1, Figs. 2 and 4).

\section{Clinoptilolite}

Clinoptilolite, a potassium-rich heulandite, was found at Sites 707 and 709 in early and middle Miocene sediments (Figs. 3 and 4) and at Site 711 in late Oligocene and early Miocene sediments (Fig. 5). It is conspicuous that the interval of clinoptilolite occurrence is barren of biogenic opal-A. If we assume a more or less similar chemical composition of the zeolites in one stratigraphic level, we can use peak intensities to obtain a quantitative estimate. With such intensity values (in detail, $\mathrm{I}_{(121) \text { barite }}$ to $\left.I_{(012) \text { corundum }} \times 100\right)$, higher clinoptilolite contents up to a ratio value of 133 (Table 1) are recorded at Site 707 (Fig. 3), whereas the lowest contents with ratio values around 15 (Table 3 ) occur at Site 711 (Fig. 5). Ratio values of around 40 (Table 2) at Site 709 (Fig. 4) fall between these two. Beside the clinoptilolite in Core 115-711A-20X of the late middle Eocene porcellanite layer at Site 711 , further clinoptilolite occurrences are restricted to the deeper stratigraphic sequence at Site 707 where several samples of chert were recovered (Paleocene to early middle Miocene; Fig. 3).

Based on SEM studies, clinoptilolite occurs mostly as single euhedral crystals of different sizes (Plate 3). Their morphology shows thick plates or laths and the monoclinic habit is often seen clearly by their coffin-shaped appearance (Plate 3, Figs. 2 and 4). The authigenic nature of the clinoptilolite is often documented in carbonate-free samples (after acetic acid treatment) by numerous molds of calcareous nannofossils (Plate 3, Figs. 1-3).

\section{Barite}

Barite was identified at all three sites, and its relative downhole variation is shown by ratio values of $I_{(121) \text { barite }}$ to $I_{(012) \text { corundum }}$ $\times 100$ (Figs. 3-5). Beside differences in the height of the values, there are several similar patterns of barite distribution at least at Sites 707 and 709 . At Site 707 ratio values of 10 are recorded in the early Oligocene sediments, followed by minimum values during the time interval from the late Oligocene to the middle Miocene (Fig. 3). In general, barite concentrations at Site 709 are highest during the Oligocene with maximum values in the early Oligocene (Fig. 4). During the early and middle Miocene, barite concentrations decrease and are distinctly enhanced during the late Miocene. Although barite concentrations at Site 711 are generally lower and are close to the limit where they are detectable by XRD, the early Oligocene maximum is also documented (Fig. 5).

In Eocene sediments there is a general trend to lower barite values with increasing age at all sites, down to values not detectable in the lower middle and early Eocene at Sites 707 and 711
(Figs. 3 and 5). Barite concentrations show good correlation to the pattern of biogenic opal occurrences in some parts of the sediments. Our SEM investigations revealed that barite occurs as euhedral crystals of rather uniform small size $(1-3 \mathrm{~mm})$ in Leg 115 sediments. The microcrystals are predominantly of granular shape (Plate 4, Fig. 3) and seem to be concentrated sometimes at various places in the sediments (e.g., within radiolarian tests).

\section{DISCUSSION}

\section{Opal Dissolution Structures}

The corrosion structures on sponge spicules revealed in the lower and middle Miocene section at Site 707 (Plate 1) and to small amounts at Site 709 are totally different from the general dissolution marks. Although different sizes of pits and holes are developed, which may be related to different penetration depths of solution, the largest pits or holes penetrate deep into the skeletons and are more or less of the same diameter between 8 and $10 \mu \mathrm{m}$. The sharp edges of the circular corrosion pits demonstrate that no homogeneous surface dissolution occurred, and the distinct corrosion pits and holes could be caused by spotshaped dissolution. Such corrosion, which is not yet understood in opaline material and could be initiated in special microenvironments, is probably caused by microbiological activity.

Within the marine environment, microbial degradation, as well as microbial construction of particle sediment components, seems to be important during several processes of early diagenesis; for example, during phosphorite genesis (Soudry, 1987), decomposition of organic matter (Berner, 1985), or manganese precipitation (Krumbein, 1983). Krumbein (1983) has summarized the problems concerning degradation of silicon components by microbial activity. After this author, it has not been completely resolved if direct microbial attack can cause the breakdown of amorphous silica (like glass) or other silicate minerals during the interaction of silicates and microorganisms. Krumbein (1983) gives several references on the microbial attack on silicates, but he also mentioned that none of these authors give clear evidence of microbial solubilization.

Similar corrosion marks in sponge spicules are described in freshwater sponges from the middle Eocene oil shales of Messel (Martini and Rietschel, 1978). Martini and Rietschel (1978) mentioned that microbial activity could contribute to the strongly developed corrosion marks. Unfortunately, the direct structures of bacteria or other microorganisms are neither preserved in the corrosion marks of the sponge spicules from the Messel-shales nor in the sediments of Site 707. Therefore, a microbial contribution to the sponge spicule corrosion cannot be proved. However, the appearance of the corrosion structures, which are restricted to distinct sediment levels, may represent a special diagenetic environment, and corrosion pits themselves may be influenced by microbial activity. The process of opal degradation is not known, and the peculiar corrosion structures may represent an endolithic boring pattern or several spot-shaped solution fronts resulted from microbial colonies. A pressure derived solution caused by grain-to-grain contact can be neglected because of the shape and configuration of the pits in the sponge spicules (Plate 1, Fig. 2).

\section{Barite}

The occurrence of barium in deep-sea sediments is associated with (1) hydrothermal activity (Arrhenius and Bonatti, 1965; Boström et al., 1973; Church, 1979) and (2) high biologic productivity in equatorial regions (e.g., Schmitz, 1987). Problems still exist in distinguishing the origin of barium in sediments from these two sources. In the Indian Ocean, there is good downcore correlation of barite and biogenic silica, proba- 
bly reflecting a common flux of both constituents to the seafloor within the equatorial zone of high productivity.

The mechanism by which biological activity causes barium to be transformed from dissolved into particulate form in the sediment is still unknown. The results of Bishop (1988) support the hypothesis of Chow and Goldberg (1960) and Church and Wolgemuth (1972) that barite precipitation occurs partly within the water column in microenvironments that are enriched in sulfate from decaying organic matter. Organically bound sulfur in diatoms is sufficient to produce particulate sulfur for the barite formation. This can be attributed to the fact that diatom frustules may form better microenvironments than other plankton groups. The remains of other siliceous organisms also appear to catalyze barite formation by adsorbing cations such as barium better than other biogenic inorganic phases (Bishop, 1988). This leads to the conclusion that conditions favoring barite formation are found in recently dead siliceous plankton and large aggregate particles, such as fecal material and marine snow (Bishop, 1988 , and references therein).

Schmitz (1987) investigated five DSDP sites in the eastern Indian Ocean between $10^{\circ} \mathrm{N}$ and $10^{\circ} \mathrm{S}$. He observed high barium concentrations in Paleogene sediments of the northernmost site (Site 217), decreasing toward younger sediments, and he observed low values in old sediments increasing in younger sediments at the southernmost site (Site 214). Schmitz (1987) explained this phenomenon by the northward drift of the Indian Plate, where high barium contents in the sediments represent the time of the position beneath the equatorial high-productivity zone. Because of the configuration of land masses around the Indian Ocean, the modern tropical divergence zone is displaced to around $10^{\circ} \mathrm{S}$ of the equator (Wyrtki, 1973).

Sites 707,709 , and 711 investigated here are located roughly between $8^{\circ} \mathrm{S}$ and $3^{\circ} \mathrm{S}$ and are therefore near the modern highproductivity center. The sites probably are not located far enough away from one another to make similar reconstructions. Nevertheless, a variance of barite distribution and also of the biogenic silica distribution with age exists at the different sites (Figs. 3-5). Maximum barite values are found at Site 707 in late to middle Miocene sediments and at Sites 709 and 711 in the lower Oligocene interval.

Because of the greater latitudinal separation between Sites 707 and 709 than between Sites 709 and 711, the difference in age is more obvious between the maximum barite values of Sites 707 and 709 than between Sites 709 and 711 . The tectonic reconstruction of the Indian Plate done by Courtillot et al. (1986) reveals that the position of Site 707 was at $25^{\circ} \mathrm{S}$ during Chron 29 (67 Ma). Since then, it has moved northward to its present position. Assuming the same latitudinal separation between Sites 707,709 , and 711 and a stable position of the high-productivity belt, Site 711 reached this zone of high productivity earlier and left it earlier than Site 707 did. This assumption fits well with the barite distribution at the sites investigated and with the findings of Schmitz (1987).

Nevertheless, a simple correlation between barite and biogenic opal does not exist that compares downhole variations in abundances (Figs. 3-5). In sediments of Eocene and older age at Sites 709 and 711 , there are low amounts of barite associated with the highest opal-A contents. In contrast, within Miocene to Pliocene sediments the correlation seems rather good.

\section{Clinoptilolite}

Beside phillipsites, clinoptilolites are another important zeolite group that was often found in deep-sea sediments from all oceans (Kastner and Stonecipher, 1978). The authigenic nature of the clinoptilolite is clearly demonstrated by the euhedral crystal shapes, an observation that was often documented (Bohrmann et al., 1989) and that was also demonstrated by various casts of sediment particles (mostly calcareous nannofossils) on clinoptilolite crystals from Leg 115 samples (Plate 3). In general, clinoptilolite was precipitated from silica-rich interstitial water if enough aluminium, alkali, and alkaline earth materials were available. Nevertheless, contrary ideas about the mineralogical precursor exist. From the Pacific deep-sea sediments, clinoptilolite formation was often regarded as an alteration product of phillipsite caused by the negative correlation of clinoptilolite to phillipsite in downhole abundances (Kastner and Stonecipher, 1978).

In contrast, a relationship between clinoptilolite formation and biogenic silica diagenesis was suggested by several authors investigating sediments from the Atlantic regions (e.g., Berger and von Rad, 1972; Nathan and Flexer, 1977; Riech and von Rad, 1979; Fenner, 1981; Thein and von Rad, 1987). In the Leg 115 sediments investigated here, a negative correlation of biogenic silica (opal-A) and clinoptilolite is clearly recorded at all sites (Figs. 3-5). Volcanic ash material was not found as an important sediment constituent, except for the distinct ash layers reported by the shipboard scientific party (Backman, Duncan, et al., 1988), and we favor a clinoptilolite genesis strongly associated with the silica diagenesis.

Given the abundance patterns within the Leg 115 sites (Figs. 3-5), clinoptilolites occur in two different levels and are associated with different mineral phases. The upper occurrence is reported in late Oligocene to early Miocene sediments at Site 711 and early-middle Miocene sediments at Sites 707 and 709. Clinoptilolite occurrences in such young sediments were not reported by Venkatarathnam and Biscaye (1973), who showed deep-sea zeolites and their variations in space and time in the Indian Ocean. Phillipsite was found in Miocene and younger sediments in the abyssal plains of the Indian Ocean.

In contrast, clinoptilolite was reported to occur in Cretaceous to Eocene sediments in areas closer to continents. At the level of zeolite occurrence in Leg 115 sediments, a steady decrease in clinoptilolite content is recorded from shallowest Site 707 in the south (Fig. 3) to deepest Site 711 (Fig. 5). Within this level, opal-A is never preserved, but sediments above and below contain opal-A, which shows a decrease in content from Sites 707 to 711 . This relationship may document that the amount of opal-A dissolution, which should have been greater at Site 711 than at Sites 709 and 707, controlled the extent of clinoptilolite formation. In this way, dissolved silica acted as a limiting factor for clinoptilolite growth.

It has often been reported that zeolites are formed predominately in clay-rich sediments (e.g., Thein and von Rad, 1987) because the opal-A to opal-CT transformation is largely retarded in contrast to carbonate-rich sediments where opal-CT precipitation is enhanced (Kastner et al., 1977). Clinoptilolite was recorded at Sites 707, 709, and 711 in carbonate-rich sediments. Furthermore, at Site 711 , clinoptilolite occurs where maximum carbonate contents were measured (Fig. 5). In the carbonate-poor sediments just above, clinoptilolite was not detected (Fig. 5). This may show that physicochemical conditions for opal-CT precipitation were not reached in the sediment level of clinoptilolite occurrence, probably because of the lower silica and higher aluminium content.

In deeper levels at Site 707, clinoptilolite precipitation occurs in Paleocene to early Eocene sediments and seems to be associated with opal-CT and authigenic quartz formation (Fig. 3). Our SEM studies show that clinoptilolite is generally the last precipitated mineral infilling fossil tests, often enclosing opal$\mathrm{CT}$ lepispheres grown during an earlier diagenetic stage. Because of opal dissolution, the interstitial water was first silica rich, and opal-CT precipitation occurred in deeper sediment sections. During opal-CT precipitation, a depletion in the silica concentration of the interstitial water was reached at one site. 
This leads to lower $\mathrm{Si} / \mathrm{Al}$ ratios in the pore water and clinoptilolite formation can start, if enough alkalies and earth alkalines are available. Such clinoptilolite formations have been described in deep-sea sediments by several authors (Berger and von Rad, 1972; Riech and von Rad, 1979; Thurow, 1988), who attributed it to a late diagenetic stage of the sediments.

\section{Silica Diagenesis}

Beside the authigenic clinoptilolite growth, opal-A was observed only in deeper levels in sediments of Eocene and older age at the three sites investigated. Diagenesis is evidenced by the macroscopic appearance of chert nodules and strongly cemented chert layers as well as by the XRD examination of such diagenetic silica minerals as opal-CT and quartz. Opal-CT and higher amounts of quartz $(>5 \%-10 \%)$ are only found in the chert nodules and chert beds but not disseminated in the sediments. Silica concretions can be divided into two different types. The first type occurred at Site 707 and at the base of Site 711 in calcareous host lithologies, where no opal-A is preserved anymore (Figs. 3-5); it is composed of various amounts of opal-CT and higher quartz contents $(10 \%-40 \%)$. In contrast, the silica concretion layer in Core 115-711-20X occurs in a small interval (3 m thick) of carbonate-free radiolarian ooze, revealing a high opalA content of $>95 \%$ (Fig. 5). It is predominantly composed of opal-CT, whereas the quartz content is not essentially higher than outside of the concretion layer (Fig. 5 and Table 3).

At nearly all sites, the first type of silica concretion, associated with calcareous sediments, seems to be concentrated to the early and early middle Eocene interval. The lack of silica concretions at Site 709 may be explained by the fact that the stratigraphic level of chert nodules was not reached at this site. This is in accordance with shipboard stratigraphic data (Backman, Duncan, et al., 1988). Chert was widely recovered by DSDP and ODP drilling activities from all oceans (e.g., Lancelot, 1973; Wise and Weaver, 1974; Keene, 1975; Hein et al., 1978; Riech and von Rad, 1979; Thein and von Rad, 1987). Their formation depends mainly on the opal-A to opal-CT transformation, which is influenced by in-situ temperature (burial depth and regional heat flow), time (maturation by aging), and chemical composition of the sedimentary facies (Riech and von Rad, 1979; Kastner et al., 1977). For most concepts of chert formation, the influence of temperature is an important factor for the opal-A to opal-CT transformation. Estimates on opal-A to opal-CT conversion temperatures are given by several authors; they range from $20^{\circ}$ to $55^{\circ} \mathrm{C}$ (Murata and Randall, 1977 : $48^{\circ} \pm 8^{\circ} \mathrm{C}$; Hein et al., 1978: $20^{\circ}-55^{\circ} \mathrm{C}$; Iijima and Tada, $1981: 22^{\circ}-33^{\circ} \mathrm{C}$; Thein and von Rad, 1987 : $25^{\circ}-50^{\circ} \mathrm{C}$ ).

Most deep-sea chert that were formed during burial of $<600$ $800 \mathrm{~m}$ are associated with carbonate-rich sediments. Their mode of formation seems to be quite different from those associated with pure siliceous rocks well described from several localities off Japan (e.g., Iijima and Tada, 1981; Tada and Iijima, 1983). In deep-sea sediments, chert associated with noncalcareous siliceous sediments as well as chert in clayey sediments are sparsely reported. Carbonate sediment components seem to play an important role during chert formation (Keene, 1975; Thein and von Rad, 1987). Such observations were supported by the experimental work of Kastner et al. (1977), who showed that the rate of opal-CT formation is accelerated by the coexistence of calcareous foraminifers.

Except for the porcellanite Sample 115-711A-20X-4, 32-37 $\mathrm{cm}$, all cemented silica layers of Sites 707 and 711 occur in carbonate-rich sediments. During burial of the sediments, opal-A was dissolved and reprecipitated as opal-CT in concretion layers and was then transformed later on to the most stable silica phase of quartz. This change from opal-A to opal-CT in the sediments takes place over small distances in the sedimentary column, resulting in a diagenetic front that was often marked by seismic reflectors (Hein et al., 1978; Thein and von Rad, 1987).

The silica concretion of Sample 115-711A-20X-4, 32-37 cm, is quite different because it occurs in a higher stratigraphic level in a 3-m-thick, carbonate-free interval. Opal-A is well preserved above and below the opal-CT cemented bed and constitutes up to $95 \%$ of the host rock lithology. This occurrence is similar to the opal-CT layers recovered by Eltanin Core 47-15 in pure diatom oozes (Weaver and Wise, 1973). This porcellanite occurred in a shallow sediment depth of $\sim 6 \mathrm{~m}$, and the host sediment is of Pliocene age. Based on the Eltanin Core 47-15 results, Wise and Weaver (1974) argued that opal-A to opal-CT transformation may occur at a very early stage in diagenesis. This is in contrast to general theories of opal-CT formation (e.g., Hein et al., 1978; Riech and von Rad, 1979; Iijima and Tada, 1981) that explain opal-A to opal-CT transformation by a later diagenetic stage occurring in much deeper sediment levels.

The opal-CT cemented rock of Eltanin Core 47-15 had long been considered to be a unique occurrence of such a young, low-buried porcellanite. Recently, new findings of similar rocks were made during Legs 113 and 120 in the Southern Ocean. During Leg 113, a Pliocene porcellanite was recovered at the top of the first core at Site 689 at the Maud Rise/Weddell Sea (Barker, Kennett, et al., 1988). At Site 751 (Leg 120), another Pliocene porcellanite was recorded at around $10 \mathrm{mbsf}$ from the Kerguelen Plateau (Schlich, Wise, et al., 1989). The youngest porcellanite (probably younger than $0.6-0.4 \mathrm{Ma}$ ) was sampled at around 7 mbsf on the Southwest Indian Ridge (Bohrmann et al., 1990). All porcellanites reported from the Southern Ocean occur in pure biosiliceous oozes and show sharp contacts with their host sediment.

The young ages of these porcellanite layers document that using geological time as a major factor for maturation, which has been postulated by most theories for silica diagenesis, is not generally requisite (e.g., Hein et al., 1978; Riech and von Rad, 1979; Kastner, 1981). However, detailed results from temperature estimations of the young porcellanites are not yet published and temperatures of $20^{\circ}-55^{\circ} \mathrm{C}$ do not seem to be reached in the sediments containing the young porcellanites (Bohrmann et al., 1990). Therefore, the young porcellanite formation in deep-sea sediments of the Southern Ocean cannot be explained by conventional concepts. Mechanisms controlling pore-water chemical composition should be considered. An early silica precipitation in relation to redox boundaries and bacterial metabolism was postulated by Zijlstra (1987) in Cretaceous chert. But up to now, no conclusive causes are found for such an unusual porcellanite formation (Bohrmann et al., 1990).

Within the Leg 115 chert, the silica concretion of Sample $115-711 \mathrm{~A}-20 \mathrm{X}-4,32-37 \mathrm{~cm}$, is quite different, and its occurrence in rather pure opaline sediments on a higher stratigraphic level shows similarities to young porcellanite occurrences from the Southern Ocean (Bohrmann et al., in press). At Site 711, carbonate-free radiolarian oozes were deposited near the middle-late Eocene boundary during two intervals caused by short fluctuations of the CCD. Within the rather pure opaline sediments, opal-CT precipitation could have occurred shortly after deposition under similar conditions, as it was reported from the young porcellanites of the Southern Ocean (Bohrmann et al., in press).

\section{CONCLUSIONS}

The study of the carbonate-free sediment portion of calcareous oozes and chalks from selected drill sites of Leg 115 re- 
vealed valuable additional information about sediment supply and silica diagenesis in the equatorial Indian Ocean. The results can be summarized as follows:

1. The abundance patterns of opal-A, quartz, clinoptilolite, and barite are similar at all sites and give valuable information for lithostratigraphic correlation.

2. During the Eocene to Oligocene, an uphole increase in carbonate content is obviously correlated with an opal-A decrease, documenting decreasing biogenic opal productivity. During Oligocene times, a decreasing succession of biogenic silica from north to south is well documented and is probably caused by the northward plate motion. Because of this plate movement, northernmost Site 711 left the zone of high biogenic opal productivity, today situated south of the equator, earlier than southernmost Site 707. Similar conclusions may be drawn from the barite occurrence, which can also be used as a paleoproductivity indicator.

3. The formation of clinoptilolite is well documented and generally occurs in sections where little or no biogenic opal-A was found. This negative correlation shows the substitution of opal-A by clinoptilolite during diagenesis. Two modes of clinoptilolite formation occur. In sediments older than the early middle Eocene, the genesis of clinoptilolite is related to enhanced silica diagenesis and occurs as the last precipitate of a succession of different silica minerals. In late Oligocene to middle Miocene sediments, a clinoptilolite formation was found that is not related to the precipitation of other silicate minerals and that could have occurred during an earlier diagenetic stage. Clinoptilolite authigenesis existed at a distinct level that showed decreasing contents from south to north, probably caused by different silica and aluminium supply within the pore water.

4. Special biogenic-opal dissolution features were observed on sponge spicules, which were probably influenced by microbiological activities in the sediments.

5. Silica concretions at Sites 707 and 711 are of two different types: (1) in calcareous host sediments without opal-A preservation in deeper sediment sections, and (2) in carbonate-free radiolarian oozes of shallow depth. The formation of Type 1 concretions fits well with generally accepted ideas of opal-A $\rightarrow$ opal-CT $\rightarrow$ quartz transformation as a time-, temperature-, and facies-related diagenetic process occurring in calcareous sediments at greater depths. Silica concretions of Type 2 do not fit into these concepts of chert diagenesis and were probably formed during an earlier diagenetic stage.

\section{ACKNOWLEDGMENTS}

We appreciate sampling help by the shipboard scientific party, especially M. T. Hurley on board JOIDES Resolution. We thank C. Samtleben and W. Reimann for operating the SEM and U. Schuldt for photographic work. A. Voß-Henneke kindly corrected the English of this manuscript. The manuscript has been greatly improved by the comments of E. Suess and the reviewers. Financial support was provided by the "Deutsche Forschungsgemeinschaft" (Grant No. TH 200-3). This contribution is No. 269 of the Alfred-Wegener Institute for Polar and Marine Research.

\section{REFERENCES}

Arrhenius, G., and Bonatti, E., 1965. Neptunism and vulcanism in the ocean. In Sears, M. (Ed.), Progress in Oceanography: London (Pergamon Press), 3:7-22.

Backman, J., Duncan, R. A., et al., 1988. Proc. ODP, Init. Repts., 115: College Station, TX (Ocean Drilling Program).

Barker, P. F., Kennett, J. P., et al., 1988. Proc. ODP, Init. Repts., 113: College Station, TX (Ocean Drilling Program).
Berger, W. H., 1976. Biogenous deep sea sediments: production, preservation, and interpretation. In Riley, J. P., and Chester, R. (Eds.), Chemical Oceanography (Vol. 5): London (Academic Press), 265-328.

Berger, W. H., and von Rad, U., 1972. Cretaceous and Cenozoic sediments from the Atlantic Ocean. In Hayes, D. E., Pimm, A. C., et al., Init. Repts. DSDP, 14: Washington (U.S. Govt. Printing Office), 787-954.

Berner, R. A., 1985. Sulphate reduction, organic matter decomposition and pyrite formation. Phil. Trans. R. Soc. London, Ser. A, 315:2538 .

Bishop, J.K.B., 1988. The barite-opal organic carbon association in oceanic particulate matter. Nature, 332:341-343.

Bohrmann, G., 1986. Accumulation of biogenic silica and opal dissolution in upper Quaternary Skagerrak sediments. Geo-Mar. Lett., 6: $165-172$.

Bohrmann, G., Kuhn, G., Abelmann, A., Gersonde, R., and Fütterer, D., 1990. A young porcellanite occurrence from the Southwest Indian Ridge. Mar. Geol.

Bohrmann, G., Stein, R., and Faugères, J.-C., 1989. Authigenic zeolites and their relation to silica diagenesis in ODP Site 661 sediments (Leg 108, eastern equatorial Atlantic). Geol. Rundsch. 89:101-114.

Boles, J. R., 1972. Composition, optical properties, cell dimension, and thermal stability of some Heulandite group zeolites. Am. Mineral., 57:1463-1493.

Boström, K., Joensuu, O., Moore, C., Boström, B., Dalziel, M., and Horowitz, A., 1973. Geochemistry of barium in pelagic sediments. Lithos, 6:159-174.

Broecker, W. S., and Peng, T.-H., 1982. Tracers in the Sea: Palisades, NY (Eldigio Press).

Calvert, S. E., 1983. Sedimentary geochemistry of silicon. In Aston, S. R. (Ed.), Silicon Geochemistry: New York (Academic Press), 143186.

Chow, T. J., and Goldberg, E. D., 1960. On the marine geochemistry of barite. Geochem. Cosmochim. Acta, 20:192-198.

Church, T. M., 1979. Marine barite. In Burns, R. G. (Ed.), Reviews in Mineralogy. Mineral Soc. Am., 6:175-209.

Church, T. M., and Wolgemuth, K., 1972. Marine barite saturation. Earth Planet. Sci Lett., 15:35-44.

Courtillot, V., Besse, J., Vandamme, D., Montigny, R., Jaeger, J.-J., and Capetta, H., 1986. Deccan flood basalts at the Cretaceous-Tertiary boundary? Earth Planet. Sci. Lett., 80:361-374.

Eisma, D., and Van der Gaast, S. J., 1971. Determination of opal in marine sediments by X-ray diffraction. Neth. J. Sea Res., 5(3):382389.

Fenner, J., 1981. Diatoms in the Eocene and Oligocene sediments off NW Africa, their stratigraphic and paleogeographic occurrences. [Ph.D. dissert.]. Univ. Kiel, Federal Republic of Germany.

Hein, J. R., Scholl, D. W., Barron, J. A., Jones, M. G., and Miller, J., 1978. Diagenesis of late Cenozoic diatomaceous deposits and formation of the bottom simulating reflector in the southern Bering Sea. Sedimentology, 25:155-181.

Iijima, A., and Tada, R., 1981. Silica diagenesis of Neogene diatomaceous and volcaniclastic sediments in northern Japan. Sedimentology, 28:185-200.

Kastner, M., 1981. Authigenic silicates in deep-sea sediments: formation and diagenesis. In Emiliani, C. (Ed.), The Sea (Vol. 7): The Oceanic Lithosphere: New York (Wiley-Interscience), 915-980.

Kastner, M., Keene, J. B., and Gieskes, J. M., 1977. Diagenesis of siliceous oozes. I. Chemical controls on the rate of opal-A to opal-CT transformation-an experimental study. Geochim. Cosmochim. Acta, 41: 1041-1059.

Kastner, M., and Stonecipher, S.A., 1978. Zeolites in pelagic sediments of the Atlantic, Pacific, and Indian oceans. In Sand, L. B., and Mumpton, F. A. (Eds.), Natural Zeolite: Occurrence, Properties, Use: New York (Pergamon Press), 199-218.

Keene, J. B., 1975. Cherts and porcellanites from the North Pacific DSDP, Leg 32. In Larson, R. L., Moberly, R., et al. Init. Repts. DSDP, 32: Washington (U.S. Govt. Printing Office), 429-507.

Kolla, V., Bé, A.W.H., and Biscaye, P. E., 1976. Calcium carbonate distribution in the surface sediments of the Indian Ocean. J. Geophys. Repts., 81(15):2605-2616.

Krumbein, W. E., 1983. Microbial Geochemistry: Oxford (Blackwell Scientific Publ.). 
Lancelot, Y., 1973. Chert and silica diagenesis in sediments from the central Pacific. In Winterer, E. L., Ewing, J. I., et al., Init. Repts. DSDP, 17: Washington (U.S. Govt. Printing Office), 377-405.

Martini, E., and Rietschel, S., 1978. Lösungserscheinungen an Schwammnadeln im Messeler Olschiefer (Mittel-Eozän). Erdöl-Erdgas-Z., 94:94-96.

Murata, K. J., and Randall, R. G., 1975. Silica mineralogy and structure of the Monterey shale, Temblor Range, California. J. Res. U.S Geol. Surv., 3:567-572.

Nathan, Y., and Flexer, A., 1977. Clinoptilolite, paragenesis and stratigraphy. Sedimentology, 24:845-855.

Riech, V., and von Rad, U., 1979. Silica diagenesis in the Atlantic Ocean: diagenetic potential and transformations. In Talwani, M. Haq, W., and Ryan, W.B.F. (Eds.), Deep Drilling Results in the Atlantic Ocean: Continental Margins and Paleoenvironment: Washing. ton (American Geophysical Union). Maurice Ewing Ser., 3:315-340.

Schlich, R., Wise, S. W., Jr., et al., 1989. Proc. ODP, Init. Repts., 120: College Station, TX (Ocean Drilling Program).

Schmitz, B., 1987. Barium, equatorial high productivity, and the northward wandering of the Indian continent. Paleoceanography, 2(1): 63-77.

Schrader, H.-J., and Schuette, G., 1981. Marine diatoms. In Emiliani, C. (Ed.), The Sea (Vol. 7): The Oceanic Lithosphere: New York (Wiley-Interscience), 1179-1232.

Soudry, D., 1987. Ultra-fine structures and genesis of the Campanian Negev high-grade phosphorites (southern Israel). Sedimentology, 34 641-660.

Tada, R., and Iijima, A., 1983. Petrology and diagenetic changes of Neogene siliceous rocks in northern Japan. J. Sediment. Petrol., 53(3): 911-930.

Thein, J., and von Rad, U., 1987. Silica diagenesis in continental rise and slope sediments off eastern North America (Sites 603 and 605, Leg 93; Sites 612 and 614, Leg 95). In Poag, C. W., Watts, A. B., et al., Init. Repts. DSDP, 95: Washington (U.S. Govt. Printing Office), 501-525.

Thurow, J., 1988. Diagenetic history of Cretaceous radiolarians, North Atlantic Ocean (ODP Leg 103, DSDP Sites 398D, 603B). In Boillot, G., Winterer, E. L., et al., Proc. ODP, Sci. Results, 103: College Station, TX (Ocean Drilling Program), 531-557.

Van Andel, T. H., 1975. Mesozoic-Cenozoic calcite compensation depth and the global distribution of calcareous sediments. Earth Planet. Sci. Lett., 26:187-194.

Venkatarathnam, K., and Biscaye, P., 1973. Deep-sea zeolites: variations in space and time in the sediments of the Indian Ocean. Mar. Geol., 15:M11-M17.

Warren, B. A., 1981. Transindian hydrographic section at Lat. $18^{\circ} \mathrm{S}$ : property distribution and circulation in the South Indian Ocean. Deep-Sea Res., 28A:759-788.

Weaver, F. M., and Wise, S. W., Jr., 1973. Early diagenesis of deep-sea bedded chert. Antarctic J., Sept.-Oct., 198-230.

Williams, L. A., and Crerar, D. A., 1985. Silica diagenesis. II, General mechanisms. J. Sediment. Petrol., 55:312-321.

Wise, S. W., Jr., and Weaver, F. M., 1974. Chertification of oceanic sediments. In Hsü, K. J., and Jenkyns, H. C. (Eds.), Pelagic Sediments: on Land and Under the Sea. Spec. Publ., Int. Assoc. Sediment., 1: 301-326.

Wyrtki, K., 1973. Physical oceanography of the Indian Ocean. In Zeitschel, B., and Gerlach, S. A. (Eds.), The Biology of the Indian Ocean: Berlin-Heidelberg-New York (Springer-Verlag), 18-36.

Zijlstra, H.J.P., 1987. Early diagenetic silica precipitation, in relation to redox boundaries and bacterial metabolism, in Late Cretaceous chalk of the Maastrichtian type locality. Geol. Minjbouw, 66:343-355.

Date of initial receipt: 11 September 1989

Date of acceptance: 22 January 1990

Ms 115B-181 


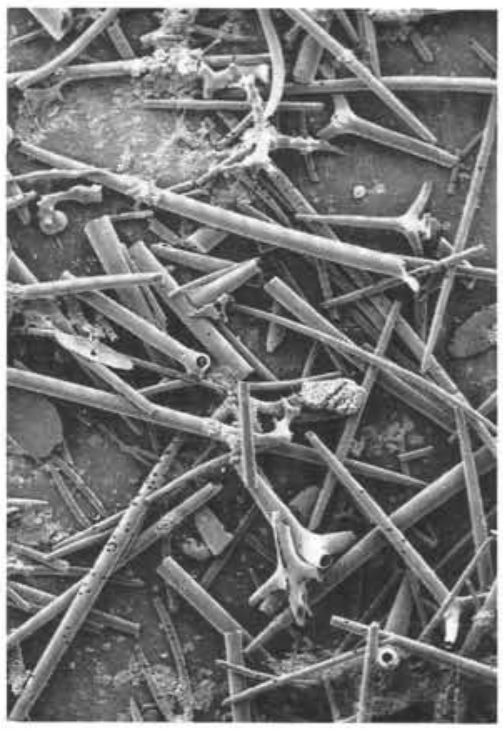

1

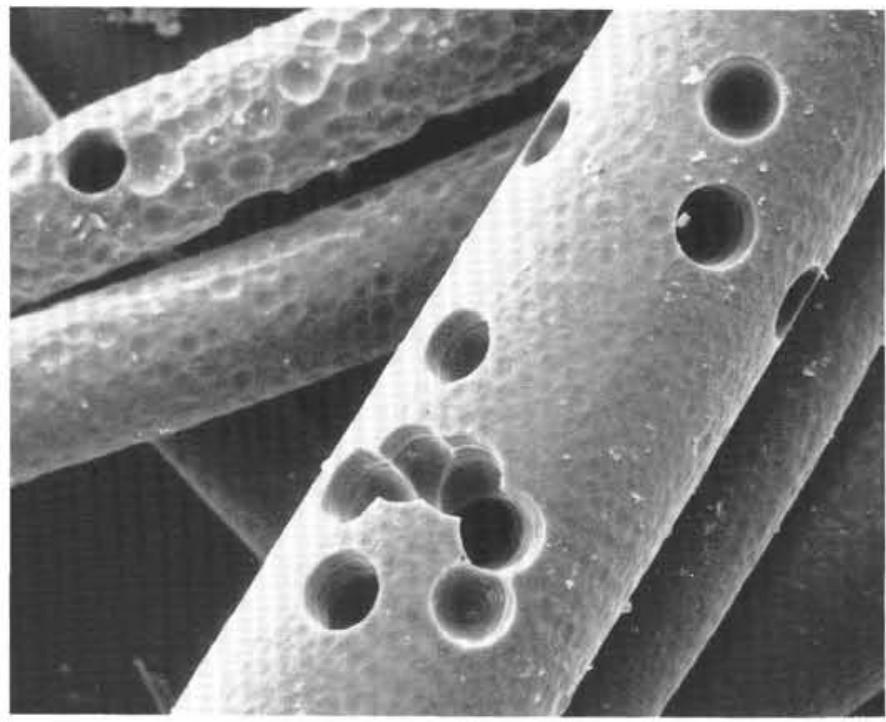

4

2
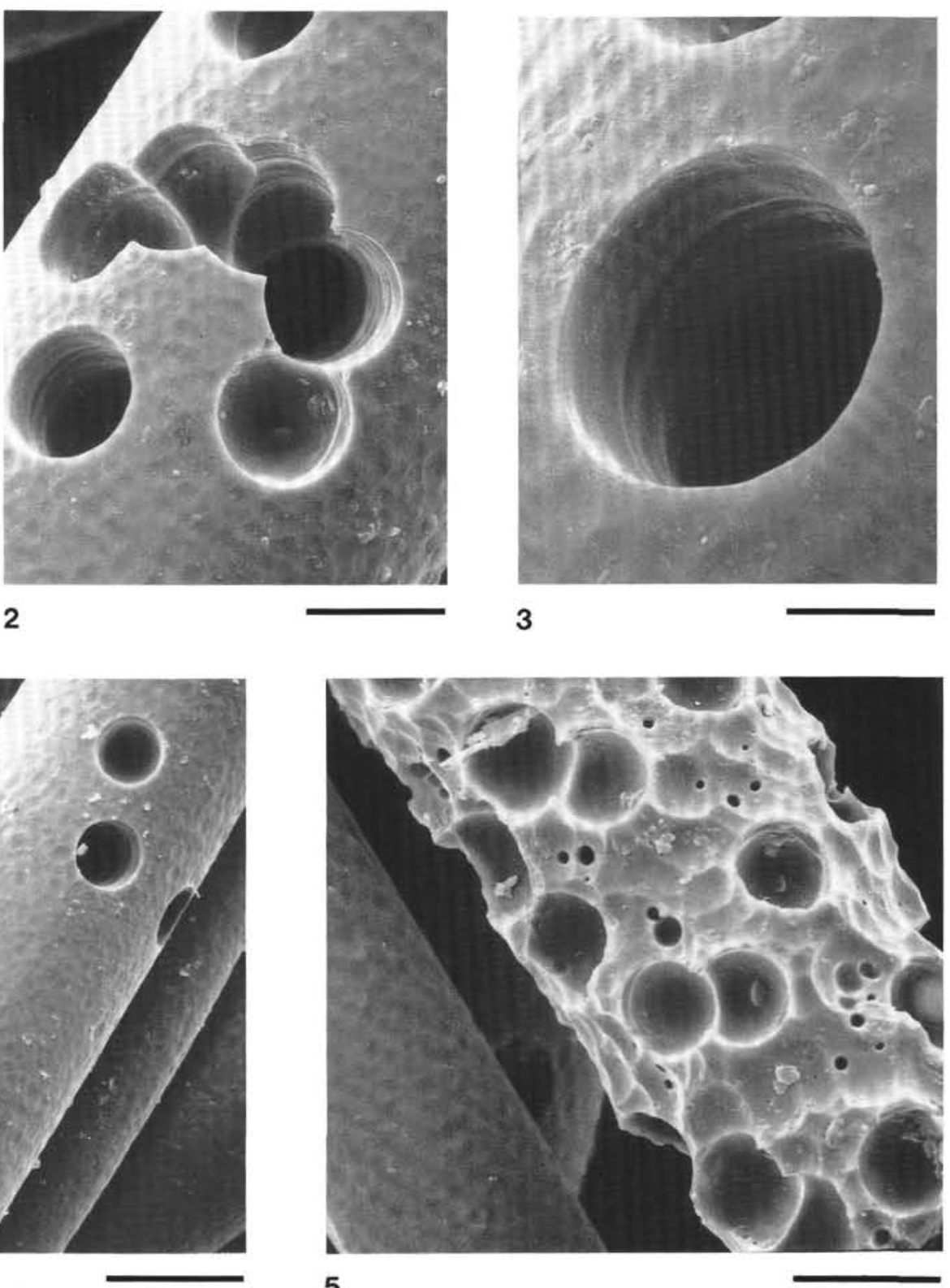

5

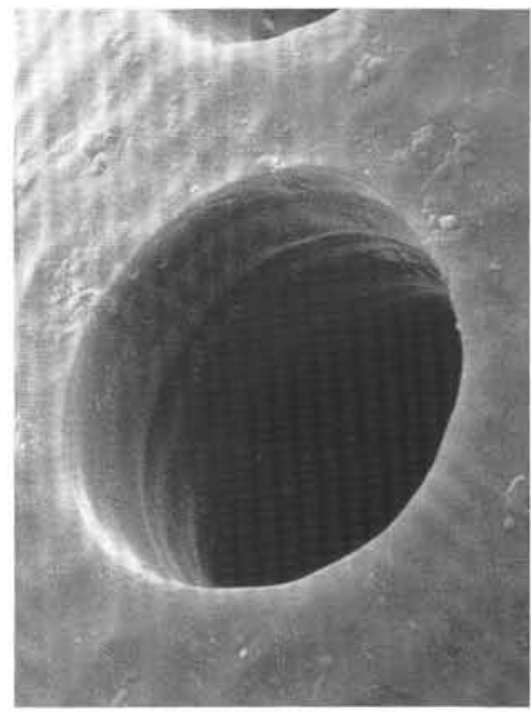

3

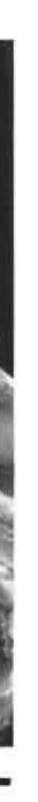

Plate 1. SEM photographs of sponge spicules from Sample 115-707B-13H-5, $100 \mathrm{~cm}$. 1. The overview of the carbonate-free sample shows the sponge spicules as the dominant constituents (scale bar $=400 \mu \mathrm{m}$ ). 2. Detail from Figure 4. Note the smooth rib structures within the corrosion pits showing probably the concentric internal structure of the opaline spicule (scale bar $=10 \mu \mathrm{m}$ ). 3. Well-rounded corrosion hole going from the surface to the axial channel. Note the smooth surface of the spicule and, by way of suggestion, a smooth honeycomb surface pattern (scale bar $=4$ $\mu \mathrm{m})$. 4. Sponge spicules with different occurrences of corrosion pits and holes (scale bar $=20 \mu \mathrm{m}$ ). 5. Strongly corroded spicule showing different sizes and penetrations of circular corrosion pits (scale bar $=10 \mu \mathrm{m}$ ). 


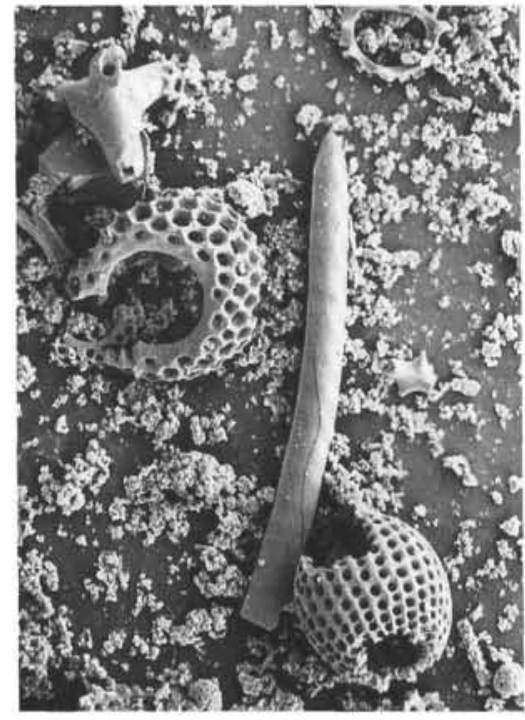

1

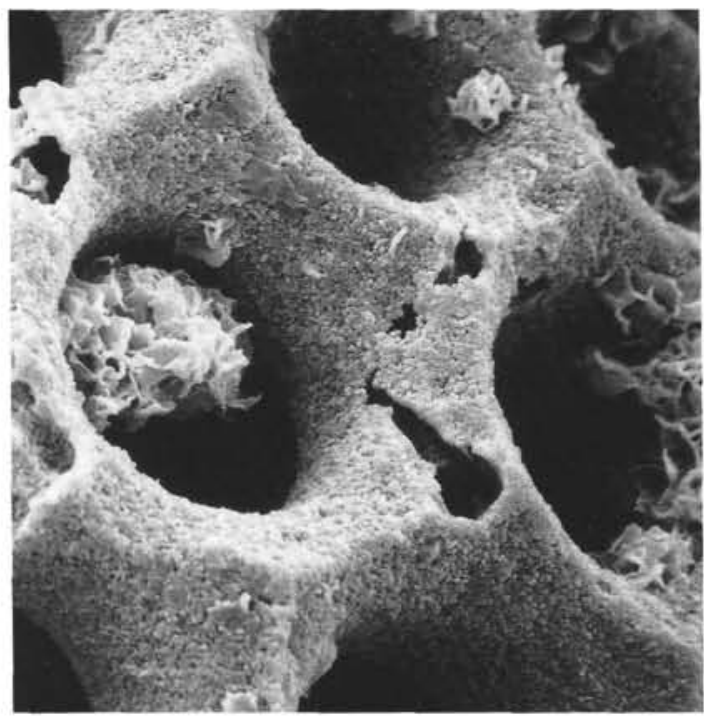

4

2
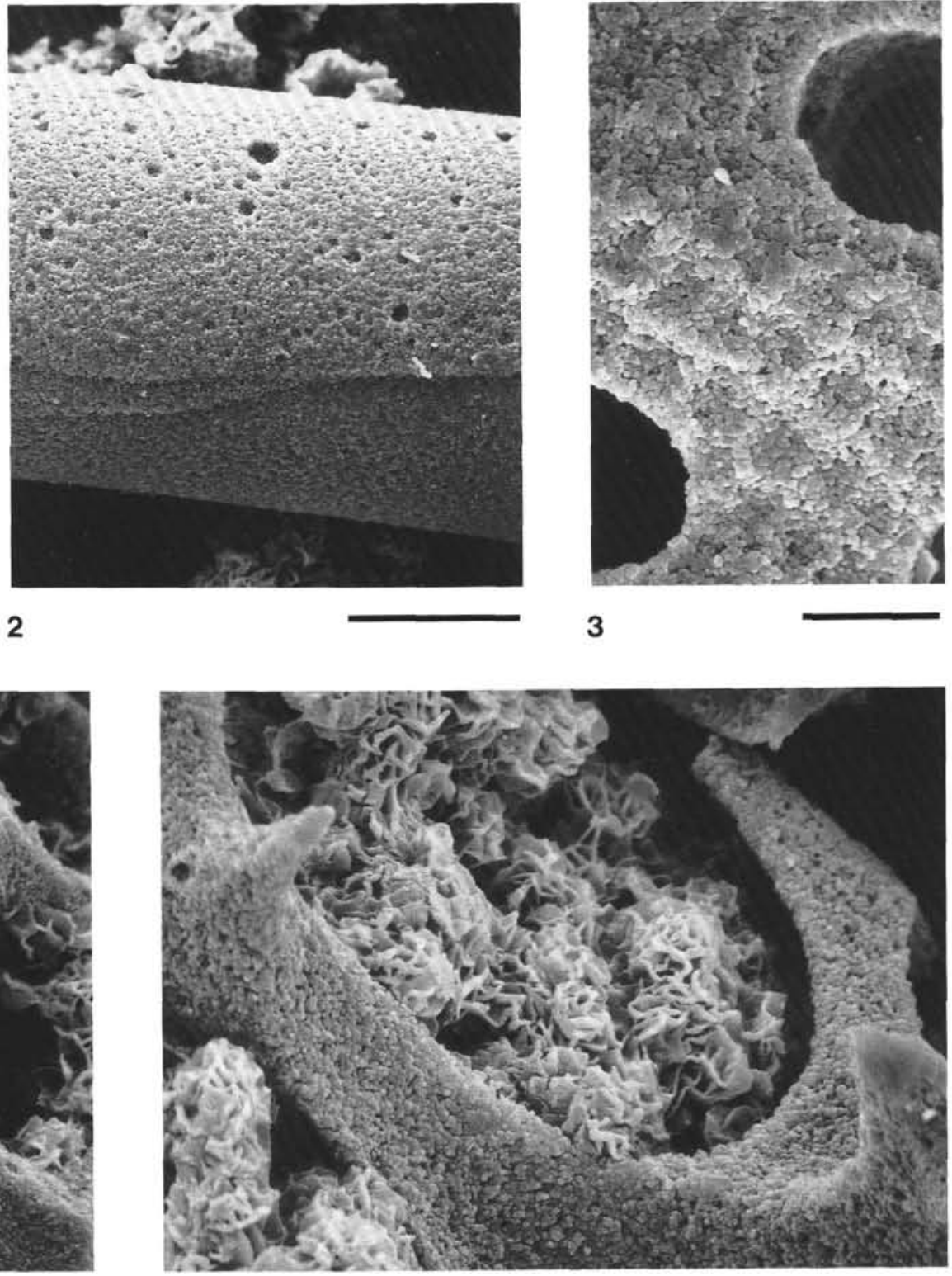

5

Plate 2. SEM photographs of opaline skeletons from Sample 115-707A-16H-5, 103-105 cm. 1. The carbonate-free sample shows numerous fragmented silica skeletons such as radiolarians and sponge spicules (scale bar $=100 \mu \mathrm{m}$ ). 2. Detail of a sponge spicule showing very rough and spongy surface including some corrosion scars (scale bar $=10 \mu \mathrm{m}$ ). 3. Detail of a radiolarian surface showing the fine globose ultrastructure of opal-A (scale bar $=4 \mu \mathrm{m}$ ). 4. Beginning of fragmentation caused by progressive dissolution of a radiolarian test (scale bar $=10 \mu \mathrm{m}$ ). 5 . Detailed view of a radiolarian filled by authigenic clay minerals (probably smectites). Because of opal dissolution the very fine globose ultrastructure of microspheres is very clear on the surface of opal-A skeleton (scale bar $=4 \mu \mathrm{m}$ ). 


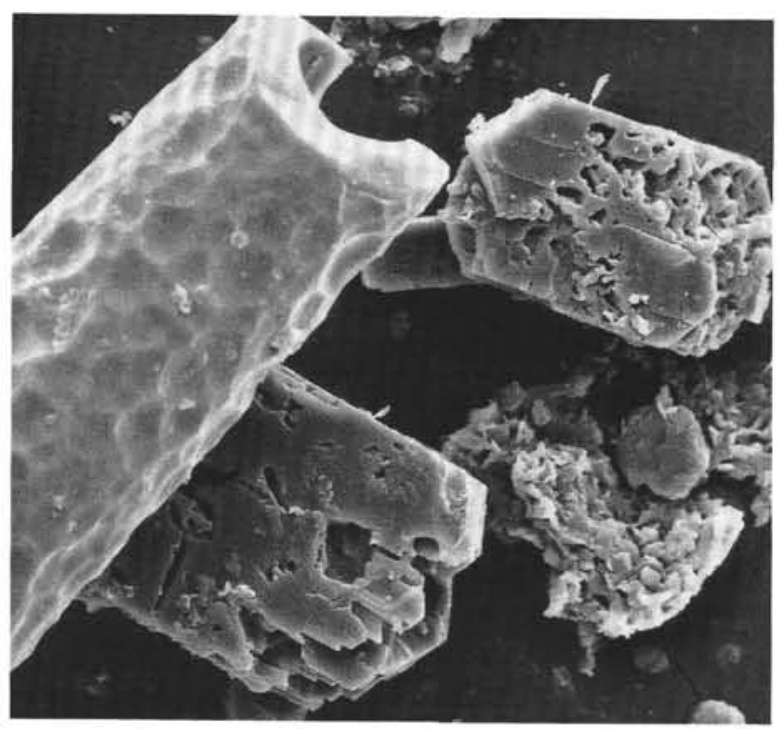

1

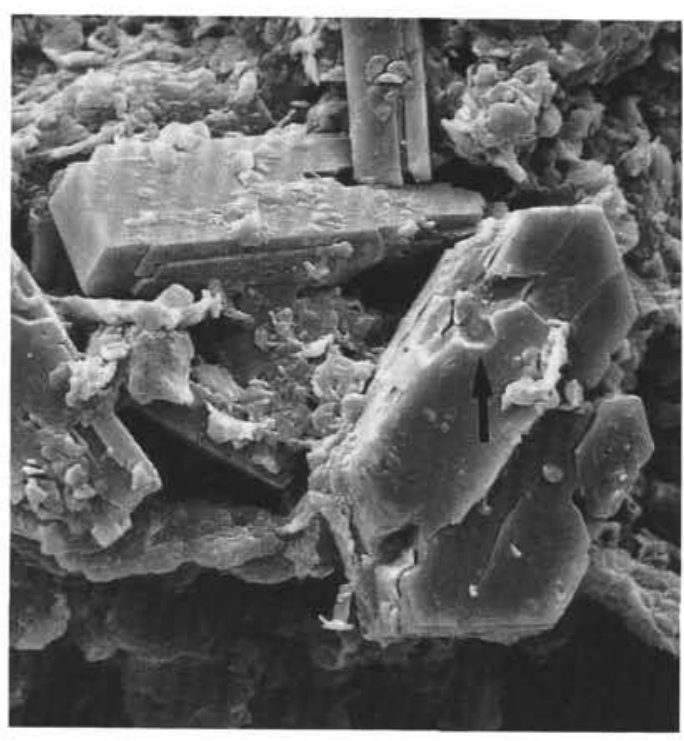

3

4

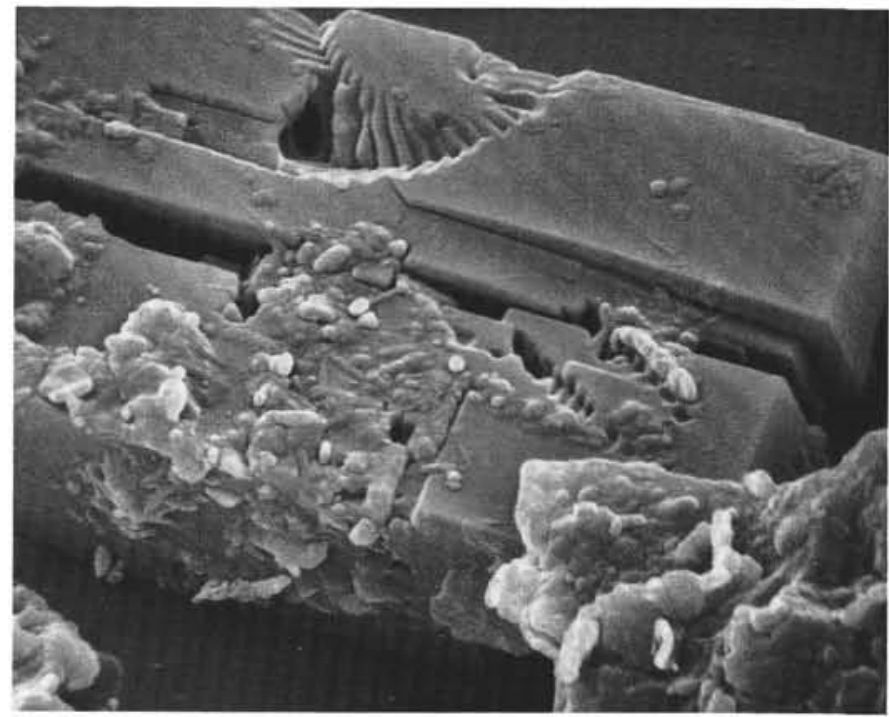

2

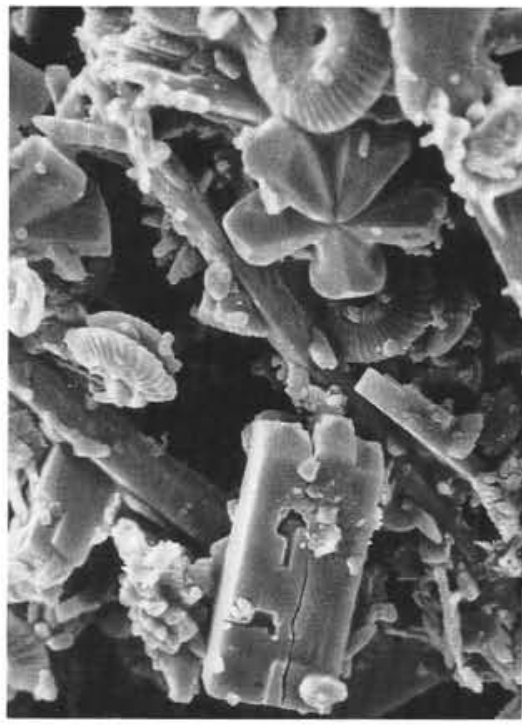

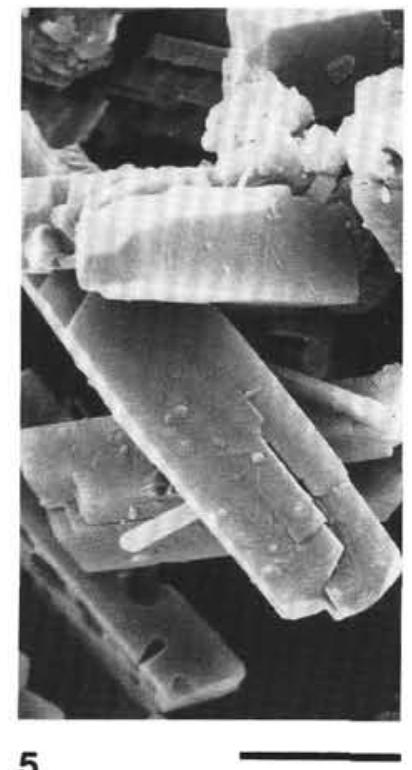

5

Plate 3. SEM photographs of clinoptilolite crystals from Sample 707B-13H-5, $100 \mathrm{~cm}$ (1 and 3), and Sample 709B-19H-6, 65-67 cm (2, 4, and 5). Carbonate particles were removed by acetic acid treatment (except in Fig. 4). 1. Strongly corroded sponge spicule and authigenic clinoptilolite crystals with numerous growth interruptions (scale bar $=10 \mu \mathrm{m}$ ). 2. Coffin-shaped clinoptilolite showing two molds from calcareous nannofossils (scale bar $=4 \mu \mathrm{m}$ ). 3. Zeolite crystals intergrown with clay minerals showing different stages in development of crystal shape. Note (arrow) the mold of a Discoaster (scale bar $=10 \mu \mathrm{m}$ ). 4. The untreated sediment sample shows the occurrence of clinoptilolite crystals in a matrix that is dominated by calcareous nannofossils (scale bar $=100 \mu \mathrm{m}$ ). 5 . Laths of clinoptilolite partly coated by very fine mineral fibers $(\mathrm{scale}$ bar $=4 \mu \mathrm{m}$ ) 

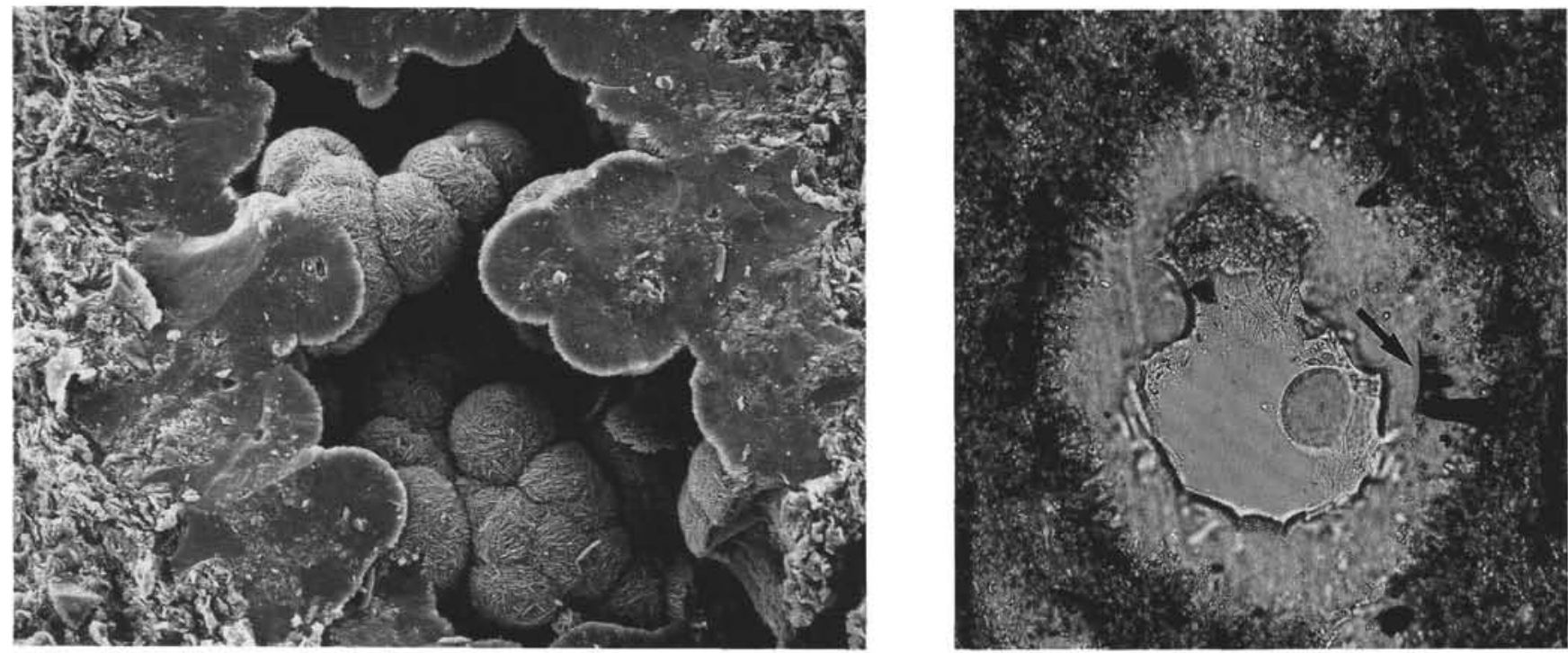

1

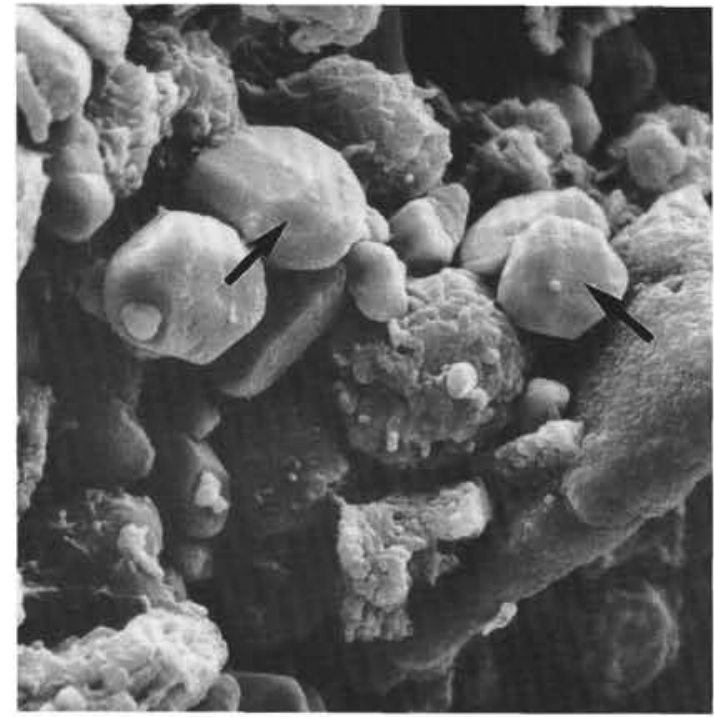

3

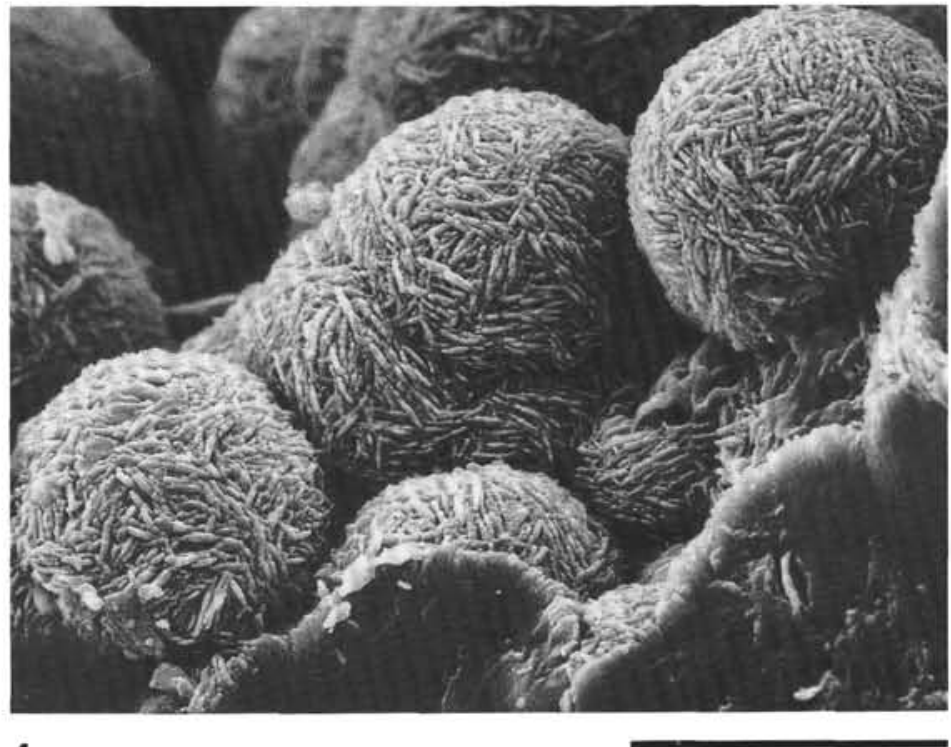

4

Plate 4. SEM photographs from Sample 115-711A-20X-4, 29-37 cm (1 and 4), and Sample 115-711A-19X-2, 41-46 cm (3); thin section photograph from Sample 115-711A-20X-4, 29-37 cm (2). 1. Opal-CT-replaced radiolaria revealing large-sized lepispheres in the center of the former skeleton (scale bar $=20 \mu \mathrm{m}$ ). 2. Opal-CT-replaced radiolaria. Note the circular shape of lepispheres in the inner space of the skeleton and the partly moldic structures of pyrite (arrow) on the former opaline skeleton (scale bar $=20 \mu \mathrm{m}$ ). 3. Euhedral granular barite crystals (see arrows, EDAX: Ba, S) with clay mineral aggregates and opal-A (scale bar $=4 \mu \mathrm{m})$. 4. Well-developed opal-CT lepispheres in open pore space $(\mathrm{scale}$ bar $=10 \mu \mathrm{m})$. 


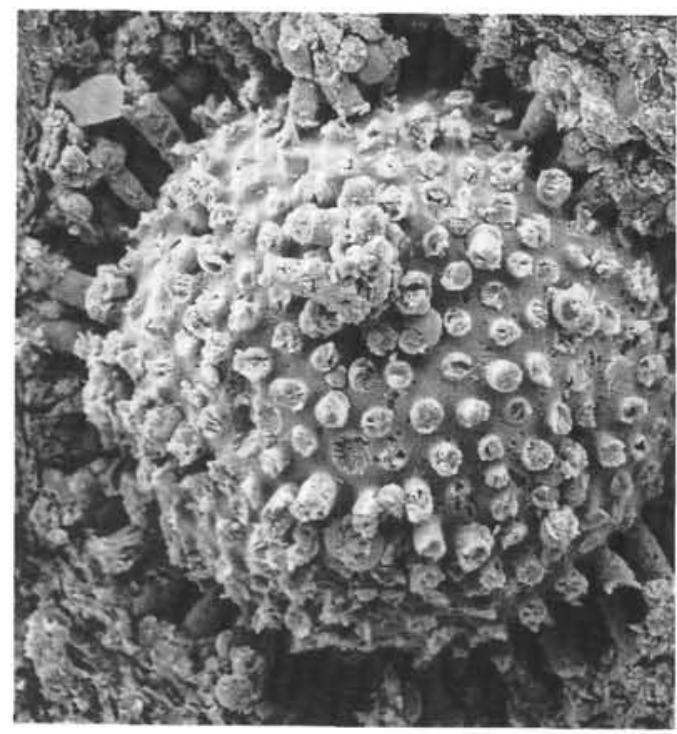

1

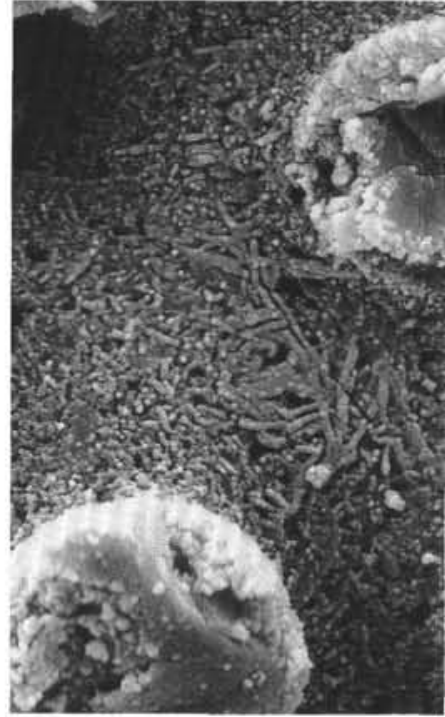

2

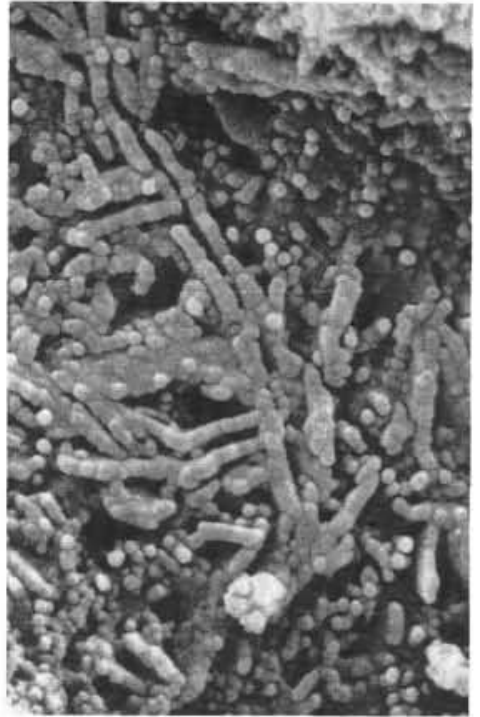

3

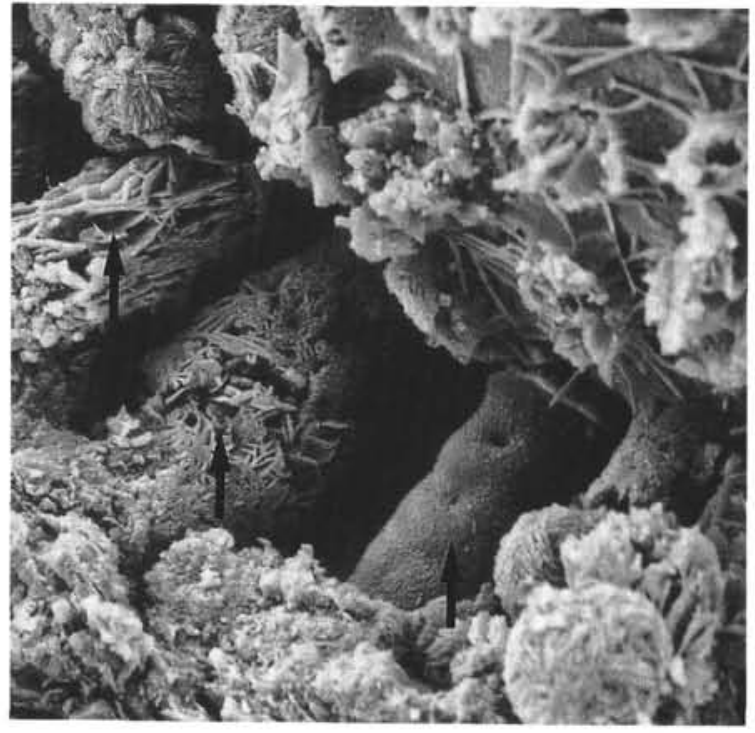

4

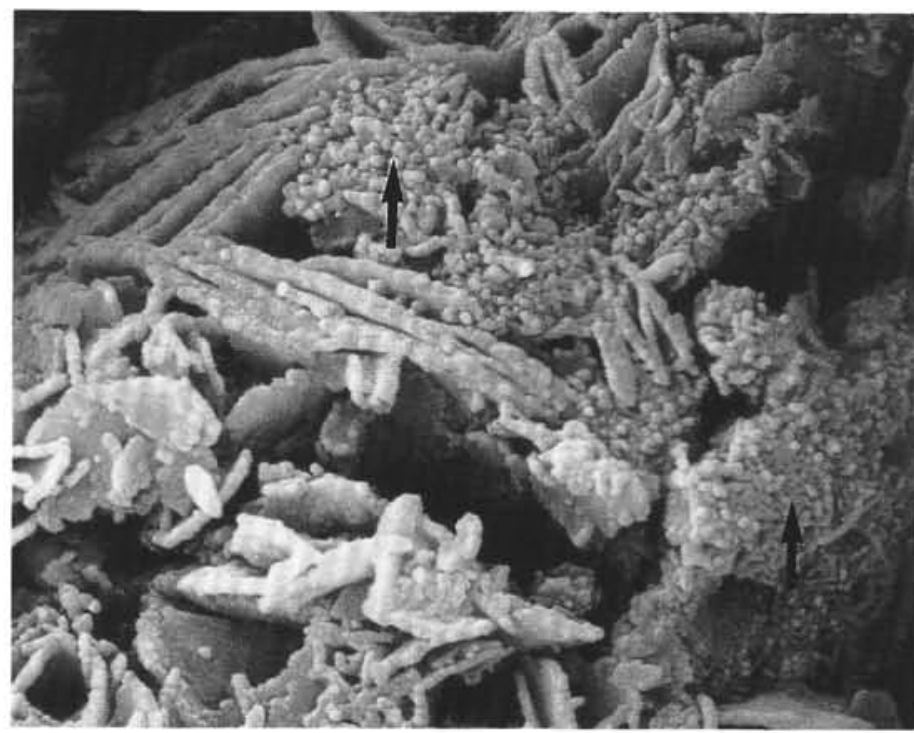

5

Plate 5. SEM photographs from porcellanite Sample 115-711A-20X-4, 29-37 cm. 1. Radiolarian skeleton mostly preserved as cast by opal-CT $($ scale bar $=40 \mu \mathrm{m})$. 2. Detail from the surface including two radiolarian spines. The originally open center of the spines seems entirely cemented by opal-CT and only a thin coat of opal-A remained. One can easily see the bladed structure of opal-CT between the two spines (scale bar $=5 \mu \mathrm{m})$. 3 . Detail of Figure 2 (scale bar $=4 \mu \mathrm{m}$ ). 4. Rim area of the radiolarian showing three spines (arrows) of different preservation stages. The right spine is still preserved as opal-A, whereas from the left spine only the opal-CT-cemented inner pore space is preserved $(\mathrm{scale}$ bar $=10 \mu \mathrm{m})$. $\mathbf{5}$. Detail from the middle spine in Figure 4. Only small parts from the opal-A-composed spine wall are preserved (arrows) and most parts show opal-CT blades (scale bar $=2 \mu \mathrm{m})$. 


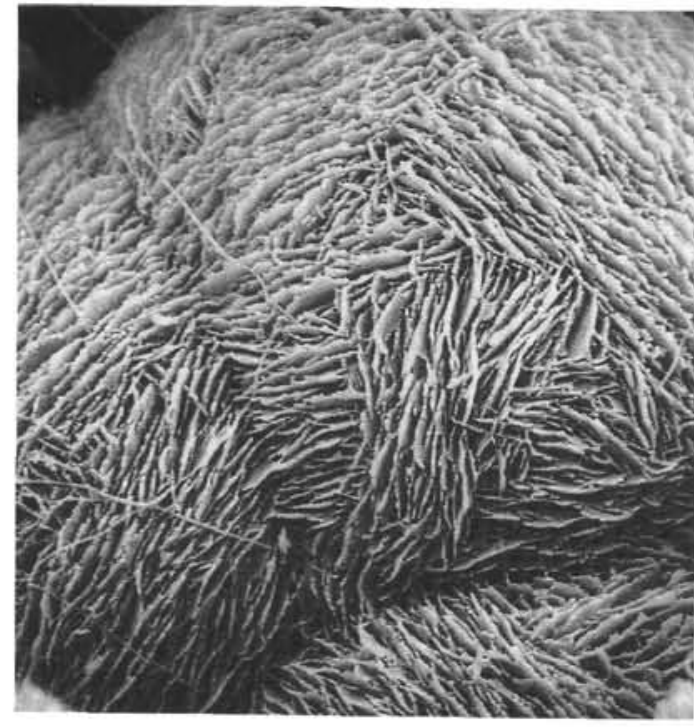

1

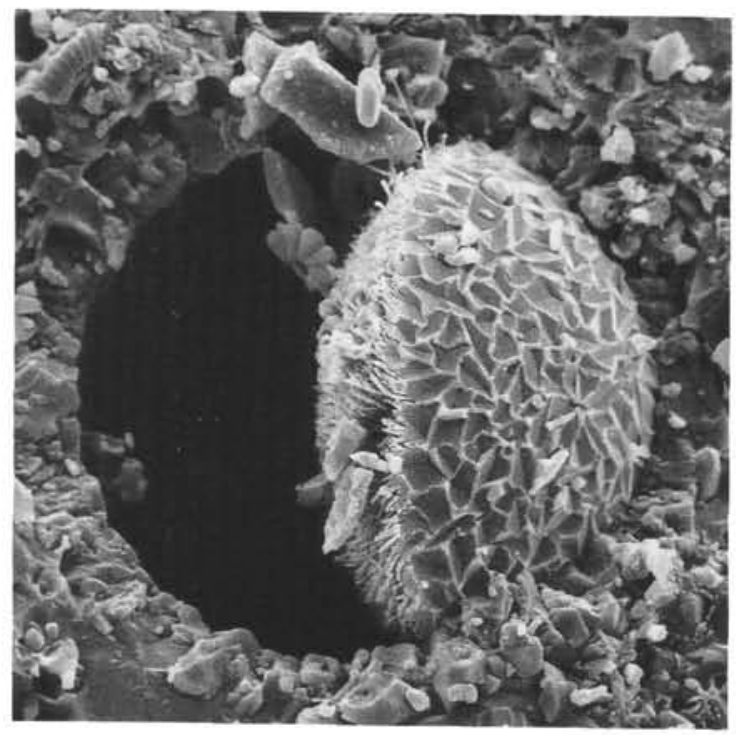

4

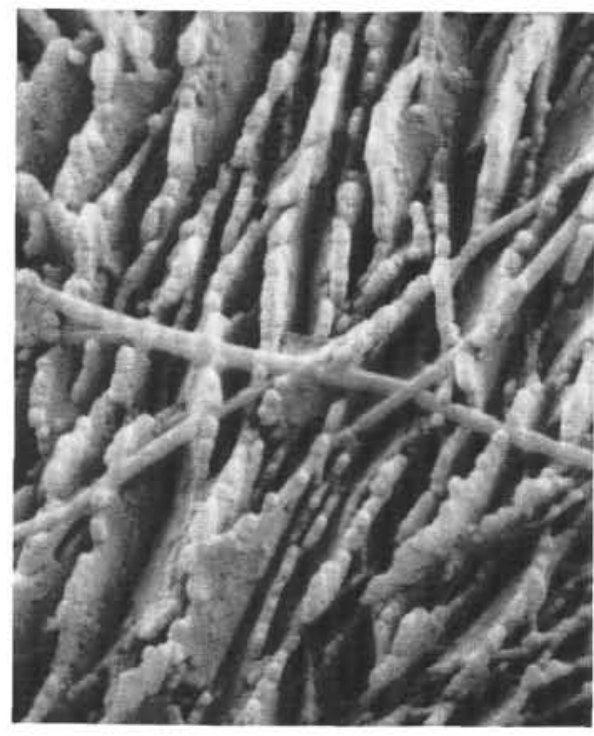

2

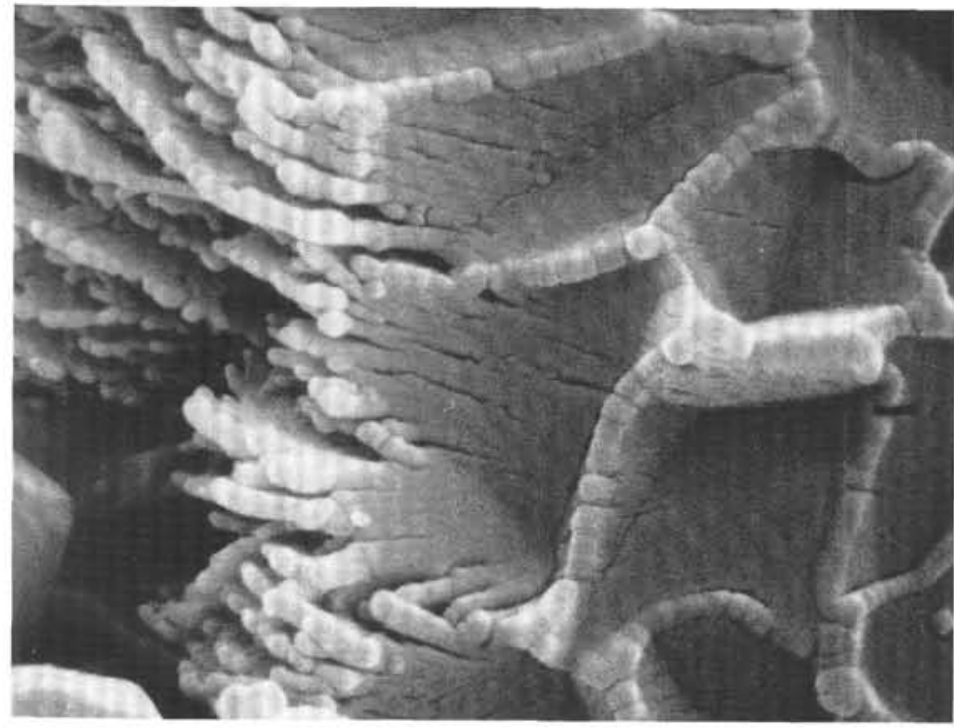

5

Plate 6. SEM photographs of opal-CT from chert Sample 115-711A-26X-CC, 30-35 cm. 1. Opal-CT lepispheres (scale bar $=10 \mu \mathrm{m})$. 2. Detail from Figure 1 showing individual opal-CT blades with irregular ragged edges (scale bar $=1 \mu \mathrm{m}$ ). 3. A detail from a fracture surface of a unbladed part of opal-CT shows a fine globose ultrastructure $($ scale bar $=1 \mu \mathrm{m})$. 4. Test of a calcareous fossil with a partly infilling of opal-CT $($ scale bar $=$ $20 \mu \mathrm{m})$. 5. Detail from Figure 4 showing the bladed structure of opal-CT only in the part of open pore space. At the right side, negatives of calcite
prisms from the fossil wall are developed (scale bar $=1 \mu \mathrm{m}$ ). 


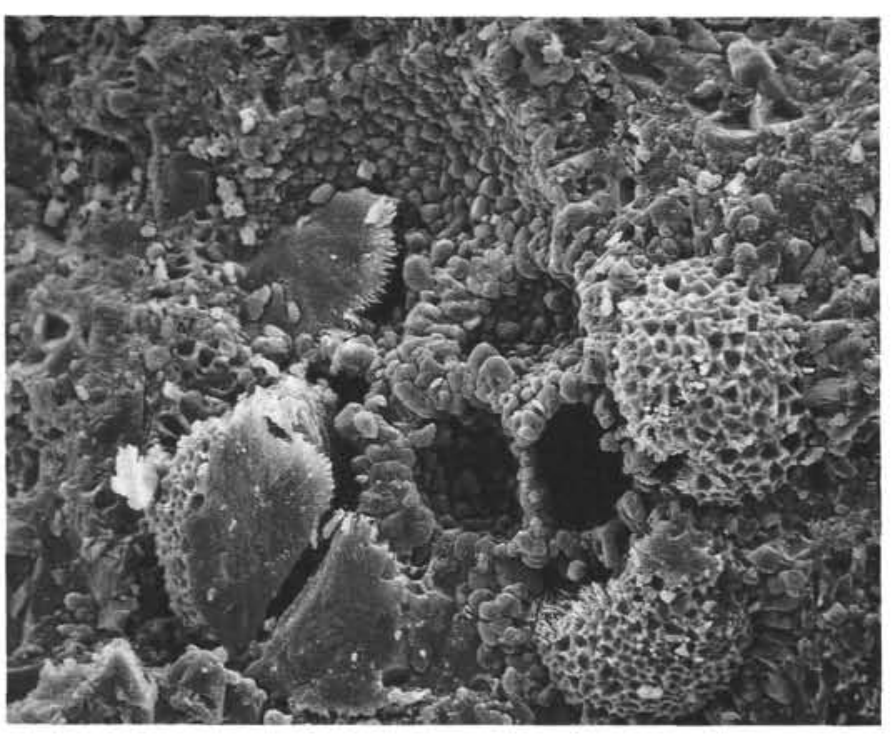

1

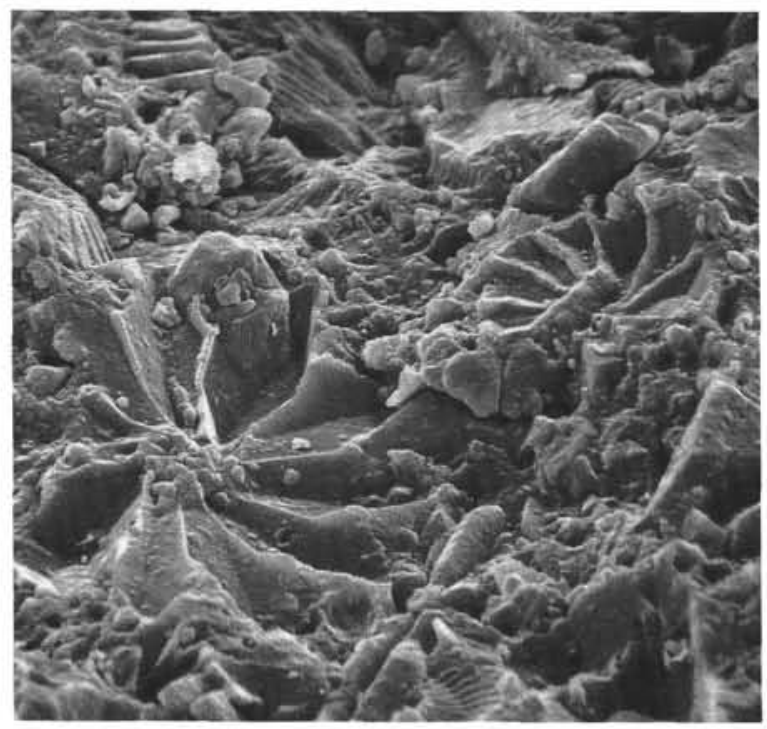

3

4

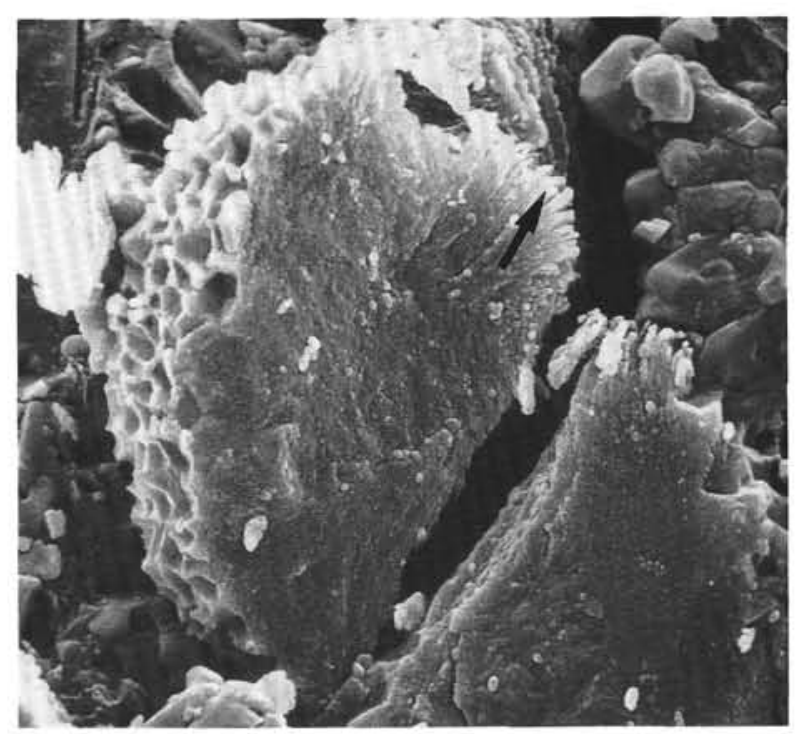

2

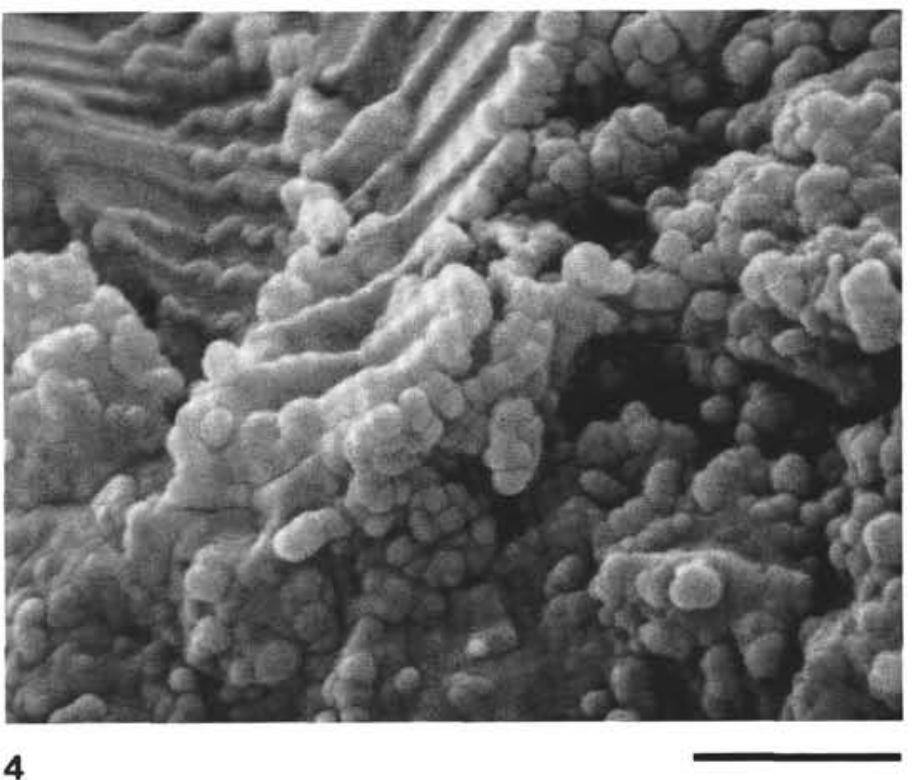

Plate 7. SEM photographs from chert Sample 115-711A-26X-CC, 30-35 cm. 1. Foraminifers embedded in a dense silica matrix. The outer chambers are filled by opal-CT (scale bar $=20 \mu \mathrm{m}$ ). 2. Detail of a chamber filling showing the negatives of calcite prisms from the chamber wall and opal-CT blades (arrow) growing into the open space of the chamber (scale bar $=10 \mu \mathrm{m}$ ). 3. Dense part of the chert showing several molds of calcareous nannofossils (scale bar $=10 \mu \mathrm{m}$ ). 4. Detail of the silica matrix composed of very fine-grained quartz forming a mold of a calcareous nannofossil (scale bar $=1 \mu \mathrm{m}$ ). 